\title{
Research Article \\ Effects of Freestream Turbulence on Cavity Tone and Sound Source
}

\author{
Hiroshi Yokoyama, Hiroshi Odawara, and Akiyoshi Iida \\ Department of Mechanical Engineering, Toyohashi University of Technology, 1-1 Hibarigaoka, Tempaku, Aichi 441-8580, Japan \\ Correspondence should be addressed to Hiroshi Yokoyama; h-yokoyama@me.tut.ac.jp
}

Received 6 July 2016; Accepted 10 November 2016

Academic Editor: William W. Liou

Copyright (C) 2016 Hiroshi Yokoyama et al. This is an open access article distributed under the Creative Commons Attribution License, which permits unrestricted use, distribution, and reproduction in any medium, provided the original work is properly cited.

\begin{abstract}
To clarify the effects of freestream turbulence on cavity tones, flow and acoustic fields were directly predicted for cavity flows with various intensities of freestream turbulence. The freestream Mach number was 0.09 and the Reynolds number based on the cavity length was $4.0 \times 10^{4}$. The depth-to-length ratio of the cavity, $D / L$, was 0.5 and 2.5 , where the acoustic resonance of a depth-mode occurs for $D / L=2.5$. The incoming boundary layer was laminar. The results for the intensity of freestream turbulence of Tu $=$ $2.3 \%$ revealed that the reduced level of cavity tones in a cavity flow with acoustic resonance $(D / L=2.5)$ was greater than that without acoustic resonance $(D / L=0.5)$. To clarify the reason for this, the sound source based on Lighthill's acoustic analogy was computed, and the contributions of the intensity and spanwise coherence of the sound source to the reduction of the cavity tone were estimated. As a result, the effects of the reduction of spanwise coherence on the cavity tone were greater in the cavity flow with acoustic resonance than in that without resonance, while the effects of the intensity were comparable for both flows.
\end{abstract}

\section{Introduction}

Flows over open cavities such as the sunroofs of automobiles and landing gear configurations of airplanes often generate self-sustained oscillations and intense tonal sound. Therefore, to reduce the tonal sound from cavity is one of the most important issues for development of rapid transport vehicles such as airplanes or automobiles.

A mechanism for oscillations with fluid-acoustic interactions in compressible cavity flows was proposed by Rossiter [1]. The vortex impinging at the downstream edge of the cavity generates acoustic waves. These acoustic waves induce vortex shedding from the upstream edge of the cavity again. Sarohia [2] measured velocity fluctuations around cavities for laminar flows over shallow cavities (depth-to-length ratio of cavities $D / L<0.35$ and freestream Mach number $M<0.07)$. He found that instability in the shear layer of the cavities was amplified by self-sustained oscillations. East [3] measured the acoustic pressure of deep cavities in the turbulent boundary layer $(1.0<D / L<8.5$ and $M<$ 0.2 ). It was clarified that the self-sustained oscillations were amplified by coupling between instability in the shear layer and the acoustic mode in the direction of cavity depth. A cavity flow without acoustic resonance does not generate selfsustained oscillations or intense tonal sound in cavity flows at low Mach numbers in the turbulent boundary layer. Recently, direct numerical simulations were performed to investigate the three-dimensional stability of cavity flows [4].

The control of self-sustained oscillations in cavity flows and sound generated from cavities have also been investigated. Huang and Zhang [5] used plasma actuators implemented at the leading edge ahead of cavities in a recent study on the control of cavity flows and obtained significantly reduced levels of tonal sound. The flow induced by actuators traversed in the streamwise direction makes the shear layer thickness nonuniform in the spanwise direction.

Lusk et al. [6] investigated the attenuation effects of steady normal mass injection from the upstream of the leading edge of cavities on oscillations in supersonic cavity flows. The blowing flows from multisegmented slots spanning the cavity width broke up large-scale structures in cavity flows and reduced the level of pressure fluctuations in the cavities. These results indicate that disturbance in the incoming boundary layer is important to reduce the cavity tone. 


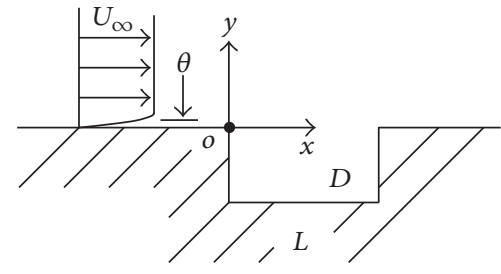

(a)

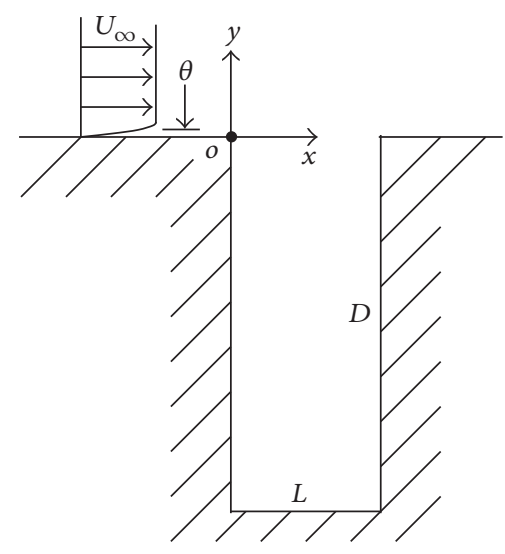

(b)

Figure 1: Configurations for flows around two-dimensional cavity. (a) $D / L=0.5$. (b) $D / L=2.5$.

TABLE 1: Computational and experimental conditions.

\begin{tabular}{|c|c|c|c|c|c|c|c|}
\hline & $D / L$ & $\mathrm{Tu}[\%]$ & $L_{e} / L$ & $\theta / L$ & $M$ & $\operatorname{Re}_{L}$ & $\operatorname{Re}_{D}$ \\
\hline \multirow{5}{*}{ Computation } & \multirow{5}{*}{0.5} & 0.0 & - & 0.0071 & \multirow{5}{*}{0.09} & \multirow{5}{*}{$4.0 \times 10^{4}$} & \multirow{5}{*}{$2.0 \times 10$} \\
\hline & & 0.9 & 0.095 & 0.0072 & & & \\
\hline & & 1.5 & 0.078 & 0.0074 & & & \\
\hline & & 1.8 & 0.090 & 0.0077 & & & \\
\hline & & 2.3 & 0.111 & 0.0078 & & & \\
\hline \multirow{5}{*}{ Computation } & \multirow{5}{*}{2.5} & 0.0 & - & 0.0071 & \multirow{5}{*}{0.09} & \multirow{5}{*}{$4.0 \times 10^{4}$} & \multirow{5}{*}{$9.9 \times 10^{4}$} \\
\hline & & 0.9 & 0.095 & 0.0072 & & & \\
\hline & & 1.5 & 0.078 & 0.0074 & & & \\
\hline & & 1.8 & 0.090 & 0.0077 & & & \\
\hline & & 2.3 & 0.111 & 0.0078 & & & \\
\hline \multirow{2}{*}{ Experiment } & 0.5 & \multirow{2}{*}{0.6} & \multirow{2}{*}{0.165} & \multirow{2}{*}{0.0072} & \multirow{2}{*}{0.09} & \multirow{2}{*}{$4.0 \times 10^{4}$} & $2.0 \times 10^{4}$ \\
\hline & 2.5 & & & & & & $9.9 \times 10^{4}$ \\
\hline
\end{tabular}

The disturbances in the boundary layer are also possibly caused by freestream turbulence, freestream vortices, and acoustic waves, as reviewed by Saric et al. [7]. Brandt et al. [8] studied the effects of the integral scale of freestream turbulence on bypass transitions of the boundary layer by using direct numerical simulations. They found that transitions occurred earlier for larger-scale freestream turbulence. The condition of the incoming boundary layer affects the cavity tone, and this could cause the past measured scatter of sound pressure spectra of cavity tone particularly at a low Mach number [9]. However, the effects of freestream turbulence on cavity flows and generated sound have not yet been clarified.

Also, it has been considered to be important to clarify the structures of the sound source to understand the mechanism for reducing the cavity tone. Larsson et al. [10] directly simulated two-dimensional flows and acoustic fields and estimated the sound source based on the Curle's acoustic analogy [11] by using fluctuating surface pressure. They found that the sound source of the cavity tone was maximal at the downstream edge of the cavity. Ask and Davidson [12] discussed the cancellation of the sources at the cavity bottom and downstream wall. However, the effects of the intensity of the Lighthill's acoustic source [13] and spanwise coherence of the source on the sound have not been investigated.

The present study directly simulated flow and acoustic fields for cavity flows with various intensities of freestream turbulence. The sound source of the cavity tone based on Lighthill's acoustic analogy [13] was estimated to clarify the reduction mechanism for the cavity tone by freestream turbulence. The effects of the weakening of the intensity of the sound source and the lowering of spanwise coherence of the sound source on the cavity tone were separately investigated.

\section{Flow Configurations}

Figure 1 outlines the configurations for flows around a twodimensional cavity. The origin of the coordinate system is located at the upstream edge of the cavity. The streamwise direction is the $x$-axis, the vertical direction is the $y$-axis, and the spanwise direction intersecting these two axes is the $z$ axis. The cavity length, $L$, is $20 \mathrm{~mm}$. The freestream velocity, $U_{\infty}$, is $30 \mathrm{~m} / \mathrm{s}$.

Table 1 summarizes the computational and experimental conditions. The depth-to-length ratio of the cavity is $D / L=$ 
0.5 and 2.5. In addition, acoustic resonance in the direction of cavity depth occurs in the flow over the cavity with $D / L=$ 2.5, as explained in Section 5.2. The freestream Mach number, $M \equiv U_{\infty} / c$, is 0.09 , where $c$ denotes the speed of sound. The Reynolds number based on the cavity length, $\operatorname{Re}_{L} \equiv U_{\infty} L / v$, is $4.0 \times 10^{4}$, where $v$ denotes the kinetic viscosity and that based on the cavity depth, $\operatorname{Re}_{D} \equiv U_{\infty} D / \nu$, is $2.0 \times 10^{4}$ for $D / L=0.5$ and $9.9 \times 10^{4}$ for $D / L=2.5$.

The velocity profile in the flow over the flat plate without a cavity at the streamwise position corresponding to the location of the upstream edge of the cavity with and without freestream turbulence is in good agreement with that of the laminar Blasius boundary layer. The ratio of the momentum thickness in the upstream boundary layer to the cavity length was slightly changed by freestream turbulence (turbulent intensity of $\mathrm{Tu}=0.0-2.3 \%$ ) and varied in the range of $\theta / L$ $=0.0071-0.0074$ in the computation. This was approximately the same as that in the experiment $(\theta / L=0.0072)$, where the freestream turbulence is $0.6 \%$.

The features of freestream turbulence in the present paper are described by the turbulent intensity, Tu, and the integral scale, $L_{e}$. The turbulent intensity, Tu, is calculated by using the rms values of the streamwise velocity fluctuations, $u_{\mathrm{rms}} / U_{\mathrm{\infty}}$, in $y / L=0.2-0.5$, where even the height of this lower limit is three times greater than the boundary layer thickness. The integral scale of the freestream turbulence, $L_{e}$, is calculated by using the autocorrelation function values of the streamwise velocity, $u$, in the same range of height. The intensity and integral scale are approximately constant in this range of height.

The computations were performed for turbulent intensity $\mathrm{Tu}=0.0 \%, 0.9 \%, 1.5 \%, 1.8 \%$, and $2.3 \%$, where the integral scale was $L_{e} / L=0.095$ for $\mathrm{Tu}=0.9 \%$ and $L_{e} / L=0.111$ for $\mathrm{Tu}=2.3 \%$. In addition, the ratio of the integral scale to the momentum thickness of the boundary layer, $L_{e} / \theta$, was 13.3 for $\mathrm{Tu}=0.9 \%$ and 15.5 for $\mathrm{Tu}=2.3 \%$. The turbulent intensity, $\mathrm{Tu}$, was $0.6 \%$ in the experiment, and the integral scale, $L_{e} / L$, was 0.165 .

It has been confirmed that no laminar-turbulent transitions in the boundary layer occurred with this freestream turbulence in both computations and experiments. Also, the preliminary computations for the flow over the cavity with $D / L=0.5$ for freestream turbulence of $L_{e} / L=0.078$ and 0.137 with turbulent intensity $\mathrm{Tu}=1.5 \%$ indicated that the effects of difference of the integral scale on the reduced cavity tone were within $2 \mathrm{~dB}$.

\section{Experimental Methods}

The experiments [14] were carried out using a suction-type, low-noise wind tunnel with a rectangular test section with a cross-section of $150 \mathrm{~mm} \times 75 \mathrm{~mm}$, as outlined in Figure 2 . The intensity of freestream turbulence was less than $0.6 \%$ and nonuniformity of the freestream was less than $\pm 0.1 \%$ at the freestream velocity of $30 \mathrm{~m} / \mathrm{s}$. The background noise level was $58 \mathrm{~dB}(\mathrm{~A})$ at the same velocity.

The test section was terminated in a spanwise manner by the end walls composed of acrylic plate and porous plate to visualize the flow within the cavity and suppress acoustic

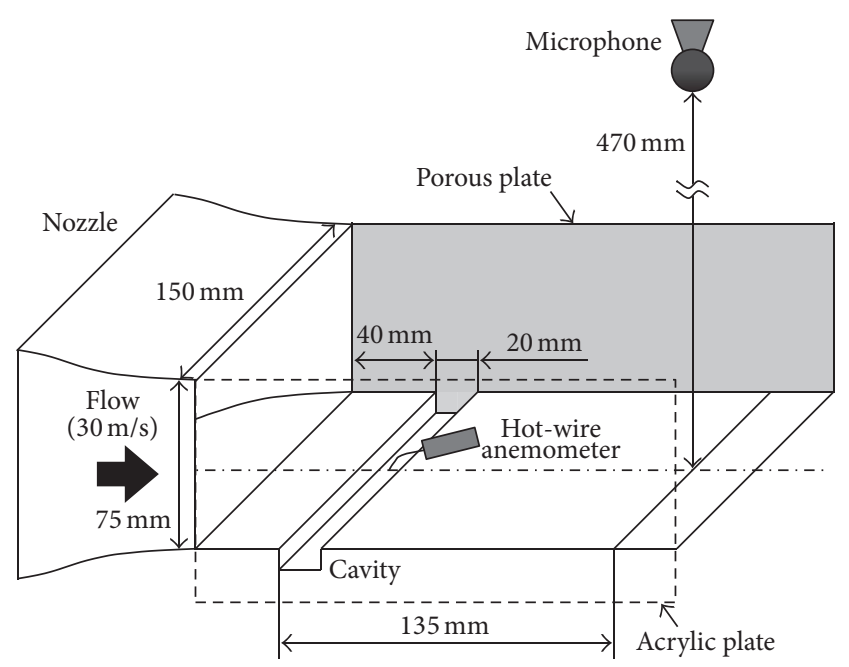

FIGURE 2: Schematics for experimental setup.

resonance in the spanwise direction. The velocity along the center of the cavity $(x / L=0.5)$ was measured using a hotwire anemometer. The acoustic pressure in the far field $(x / L$ $=6.75, y / L=23.5)$ was measured with a nondirectional $1 / 2$ inch microphone.

\section{Numerical Methods}

4.1. Governing Equations and Finite Difference Formulations. To clarify the fluid-acoustic interactions in the cavity flows, flow and acoustic fields were simulated simultaneously by directly solving the three-dimensional compressible NavierStokes equations in the conserved form.

The spatial derivatives were evaluated using a sixth-orderaccurate compact finite difference scheme [15] (fourth-orderaccurate scheme at the boundaries). The time integration was performed by the third-order-accurate Runge-Kutta method.

In order to reduce the computational cost, large-eddy simulations (LES) were performed in the present study. A tenth-order-accurate spatial filter dissipated the energy that should be transferred to subgrid scale vortices. This filter also removed numerical instabilities due to the compact finite difference scheme [16]. The details on these computational methods have been presented in Yokoyama and Kato [17].

4.2. Computational Grids. Figure 3 shows the computational grid for the flow over the cavity with $D / L=2.5$. The spanwise length of the computational domain is $W / L=1.0$ and the spanwise grid resolution is $\Delta z / L=0.0125$. It has been preliminarily confirmed that the computational results with $W / L=1.0$ approximately agree with those with a wider computational domain $(W / L=2.0)$. The computational grid for the flow over the cavity with $D / L=0.5$ was the same as that for the range of $y / L \geq-0.5$ for the flow over the cavity with $D / L=2.5$. The total grid points are 18 million.

Figure 4 outlines the computational domain that is divided into vortical, acoustic, and buffer regions. The vortical region is from the upstream edge to the downstream edge of 


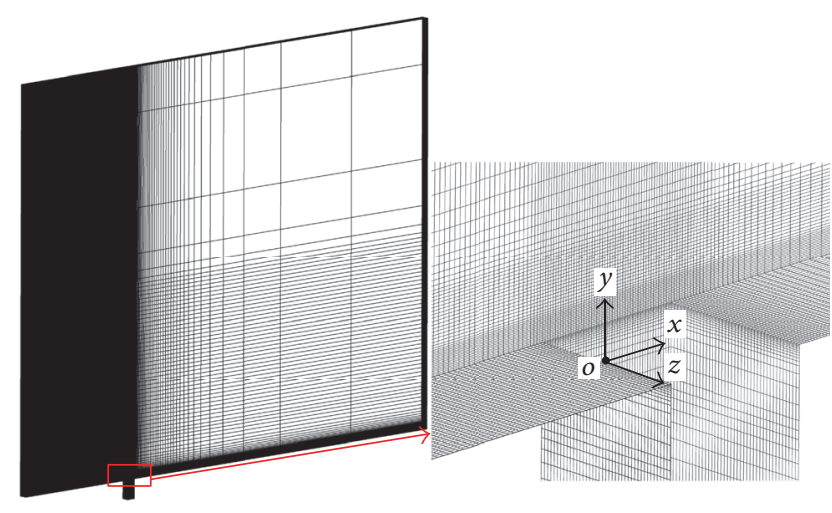

Figure 3: Computational grids for cavity flow with $D / L=2.5$ (every second grid line is shown).

the cavity $(0.0 \leq x / L \leq 1.0$ and $-0.1 \leq y / L \leq 0.1)$, where the shear layer is spread. The grid resolutions are $\Delta x / L=$ $\Delta y / L \leq 0.01$ and sufficiently fine to capture the large-scale vortical structures and active fine scale vortices in the cavity flow. The predicted velocity spectra will be discussed later in Section 5.1.

The region surrounded by the dashed line $(-12.5 \leq x / L \leq$ 8.5 and $y / L \leq 23.5)$ in Figure 4 is the acoustic region. The acoustic region has an extent that is three and two times larger than the acoustic wavelength of the fundamental frequency of the cavity tone with $D / L=0.5$ and 2.5 , respectively. More than 10 points are used per acoustic wavelength that is two and four times greater than the fundamental frequency of the cavity tone with $D / L=0.5$ and 2.5 , respectively, in the grid resolutions in the acoustic region.

In the buffer region, the grid is sufficiently stretched to dissipate acoustic and voritcal disturbances near the artificial nonreflecting boundary conditions.

As discussed in Section 5, the comparison of the predicted flow and sound with those measured clarifies that it is possible to capture acoustic waves radiating from the cavity flow by using this computational grid and these numerical methods.

4.3. Boundary Conditions. Figure 4 also shows the boundary conditions. Nonreflecting boundaries [18-20] are used at the inflow and outflow boundaries. On the lower boundary in the upstream of the cavity, the boundary condition is changed from a slip wall to a nonslip and adiabatic wall. The position of this change was determined in order to set the boundary layer thickness to be that measured. Periodic boundary conditions are used in the spanwise direction.

A uniform steady flow is asymptotically imposed in the inflow buffer region $(x / L \leq-12.5)$ for the cavity flow without freestream turbulence $(\mathrm{Tu}=0.0 \%)$. In the case with freestream turbulence, a homogeneous turbulence field is imposed on the uniform steady flow in the inflow buffer region.

This homogeneous turbulence field was separately predicted as box turbulence, where the computation starts from initial flow fields based on the von Karman spectrum [21]. These methods were described in detail in the previous

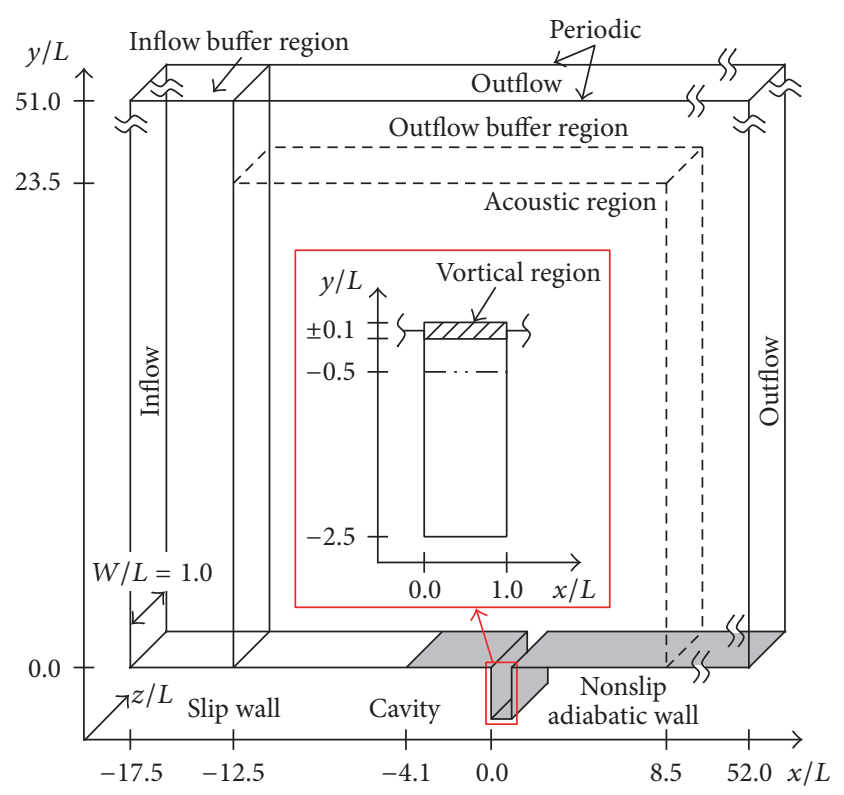

FIGURE 4: Computational domain and boundary conditions.

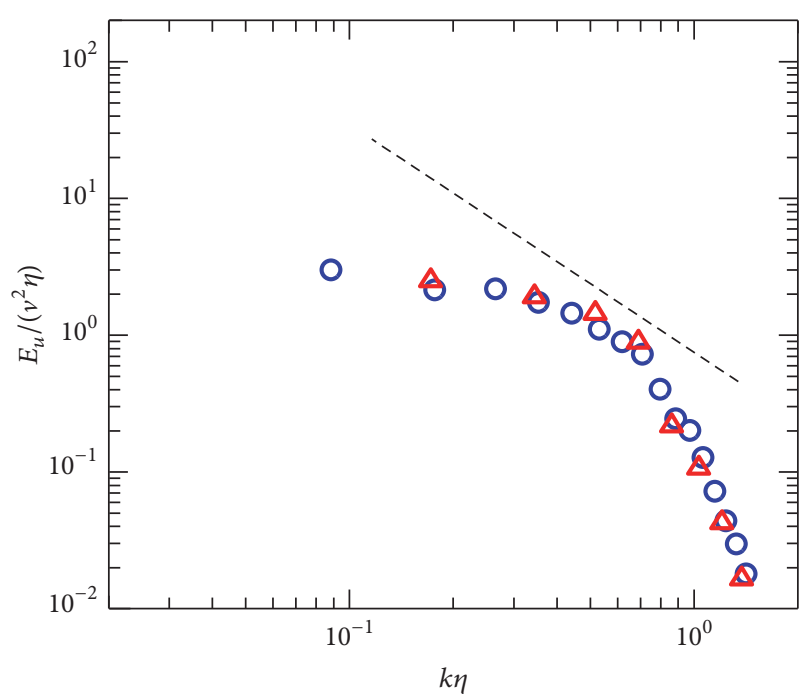

O Box turbulence for $\mathrm{Tu}=0.9 \%$

$\triangle$ Box turbulence for $\mathrm{Tu}=2.3 \%$

- - - Curve of $-5 / 3$ power

FIGURE 5: Energy spectra of homogeneous box turbulence.

paper [17]. The turbulent intensity and scale were controlled by using the parameters of the von Karman spectrum. Figure 5 plots the energy spectrum of homogeneous box turbulence. The Taylor scale of homogeneous turbulence is almost $20 \mathrm{~mm}$.

4.4. Prediction of Acoustic Far Fields. The porous Ffowcs Williams and Hawkings (FW-H) method [22-24] was used to predict the acoustic pressure at the measurement point $(x / L=6.75$ and $y / L=23.5)$ because predicting acoustic pressure in the far field with direct simulation greatly expends 


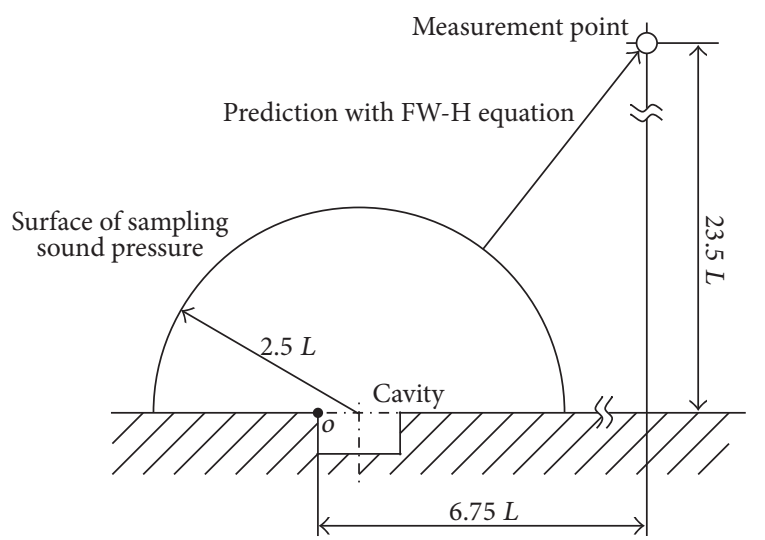

FIGURE 6: Schematics for prediction of acoustic far field by porous FW-H.

computational resources and time. The FW-H formula can be written as

$$
\begin{aligned}
L_{i j} & =p \delta_{i j} \\
4 \pi p^{\prime}\left(\mathbf{x}_{2}, t\right) & =\frac{1}{c} \frac{\partial}{\partial t} \int_{s}\left[L_{i j}\left(x_{1}, \tau\right) \frac{\widehat{\mathbf{r}} \widehat{\mathbf{n}}}{r}\right]_{\mathrm{ret}} d S\left(\mathbf{x}_{1}\right),
\end{aligned}
$$

where $\mathbf{r}=\mathbf{x}_{2}-\mathbf{x}_{1}, r=|\mathbf{r}|, \widehat{\mathbf{r}}=\mathbf{r} / r$, and $\widehat{\mathbf{n}}$ represent the outward unit normal vector of surface $S$. The subscript "ret" denotes consideration of retarded time $\tau=t-r / c$. The pressure, $p$, was sampled at the semicylindrical surface of the radius, $r=$ $2.5 L$, from the center of the cavity $(x / L=0.5$ and $y=0)$, as outlined in Figure 6.

The spanwise computational domain, $W=L$, was smaller than that of the experiment, $W_{e}=7.5 \mathrm{~L}$, to reduce computational resources. To take the effects of this difference into consideration, the sound pressure levels, $\operatorname{SPL}(f)$, were estimated by using the equivalent coherent length, $L_{c}(f)$, following (2) [25-27]:

$$
\begin{aligned}
\operatorname{SPL}(f)= & \operatorname{SPL}_{s}(f)+10 \log _{10}\left(\frac{W_{e}}{W}\right) \quad\left(L_{c}(f) \leq W\right), \\
\operatorname{SPL}(f)= & \operatorname{SPL}_{s}(f)+20 \log _{10}\left(\frac{L_{c}(f)}{W}\right) \\
& +10 \log _{10}\left(\frac{W_{e}}{L_{c}(f)}\right) \\
\operatorname{SPL}(f)= & \operatorname{SPL}_{s}(f)+20 \log _{10}\left(\frac{W_{e}}{W}\right) \quad\left(W_{c}(f) \leq W_{e}\right), \\
&
\end{aligned}
$$

where $\operatorname{SPL}_{s}(f)$ is the sound pressure level predicted with the above porous $\mathrm{FW}-\mathrm{H}$ method and the second and third terms on the right hand side are the correction terms. This length was determined by the coherence function values of the vertical velocity along the center of the cavity $(x / L=$ $0.5, y=0)$ in the spanwise direction, $\gamma^{2}(f, \Delta z)=1 / 3$.
The coherence function was computed by the same way as that in the reference by Van Der Velden et al. [28] as indicated in (3):

$$
\gamma^{2}(f, \Delta z)=\frac{\left|\Phi_{12}\left(f, z_{1}, z_{2}\right)\right|^{2}}{\left|\Phi_{11}\left(f, z_{1}, z_{2}\right)\right|\left|\Phi_{22}\left(f, z_{1}, z_{2}\right)\right|},
$$

where $\Phi_{11}$ and $\Phi_{22}$ denote autopower spectra and $\Phi_{12}$ denote the cross-power spectrum between two points along a given dimensional line $\Delta z=z_{2}-z_{1}$. The equivalent coherent length, $L_{c}(f)$, also has been confirmed to approximately agree with the distance for the phase difference in Lighthill's stress tensor at the same position of $\phi=\pi / 4$ ( 45 degrees).

When the coherence is larger than $1 / 3$ in the entire computational region, the equivalent coherent length was computed by linear extrapolation using the values in the range of $\Delta z / L=0.25-0.5$, where the gradient of the coherence is approximately constant.

\section{Validation of Computational Accuracy}

5.1. Flow Field. The predicted flow and acoustic fields for the cavity flow without freestream turbulence $(\mathrm{Tu}=0.0 \%)$ were compared with those measured because the measured turbulent intensity in the boundary layer was negligibly small.

Figure 7 shows the predicted and measured profiles of mean velocity and turbulent intensity at the center of the cavity $(x / L=0.5)$ for the cavity flow with $D / L=0.5$, where the contributions of vertical velocity $v$ to the velocity measured by a hot-wire anemometer were considered as $u_{h}=$ $\left(u^{2}+(0.5 v)^{2}\right)^{0.5}$ in the same way as our previous research [27]. It should be noted that the markers in Figure 7 in the predicted results represent only data to sample in the computational duration time, where the grid resolutions are finer. It is clarified that the predicted results agree well with those measured.

Figure 8 shows the predicted and measured power spectra of the velocity, $u_{h} / U_{\infty}$, at the center of the cavity and the height for the maximal turbulent intensity $(x / L=0.5$ and $y / L=0.02$ ) for the cavity flow with $D / L=0.5$. The frequency resolution of all spectral analyses is $\Delta \mathrm{St} \equiv \Delta f L / U_{0}=$ 0.104 . The predicted spectrum was averaged 20 times, where the samples were overlapped by $50 \%$ and the duration time of the samples for spectral analysis was $100 L / U_{0}$. Also, the experimental spectrum was averaged 9400 times with respect to time.

As shown in Figure 8, there is the main peak of $\mathrm{St}_{f}=1.56$ (fundamental frequency) in both predicted and measured results, where the cavity tone radiates at this frequency. Moreover, the predicted power at the fundamental frequency is in good agreement with that measured.

To clarify the effects of the width of the computational domain, $W$, on the predicted flow fields, the coherence of the normal velocity at $x / L=0.5, y=0$ was computed for the flow fields predicted with different domains of $W / L=$ 1.0 and 2.0. Figure 9 shows the predicted coherence at the fundamental frequency. It is presented that the distributions of the coherence predicted with the original width are in good agreement with those with the double width. This means 


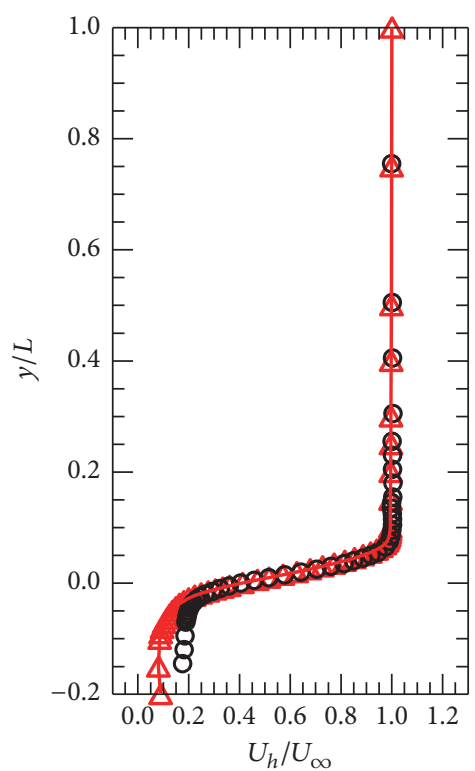

$\triangle$ Comp.

O Exp.

(a)

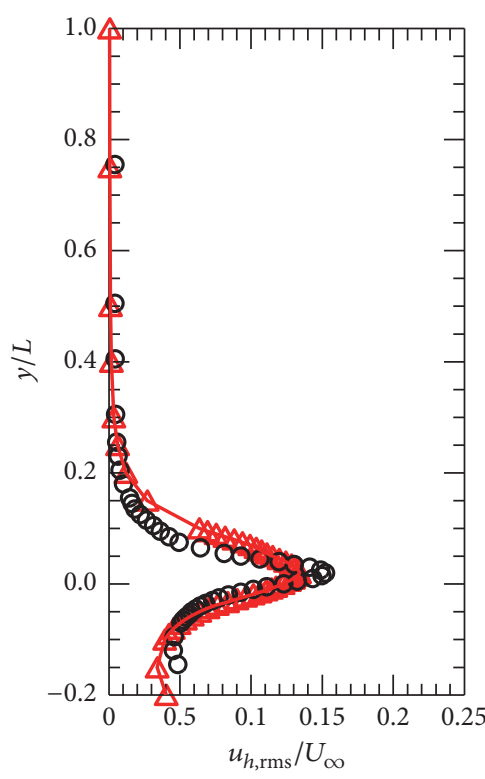

$\triangle$ Comp.

O Exp.

(b)

FIGURE 7: Predicted and measured profiles of mean velocity (a) and turbulent intensity (b) for the cavity flow with $D / L=0.5$.

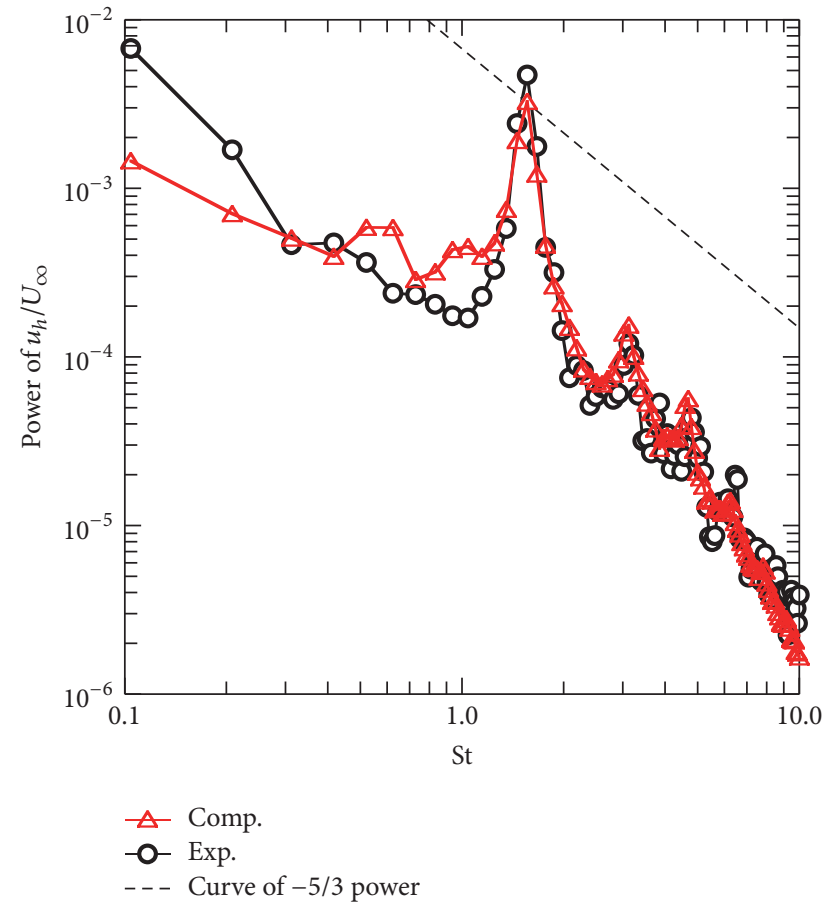

FIGURE 8: Predicted and measured power spectra of velocity fluctuations $(x / L=0.5$ and $y / L=0.02)$ for cavity flow with $D / L=$ 0.5 .

that the independency of the computational results on the width of the domain supports the adoption of the periodic conditions.

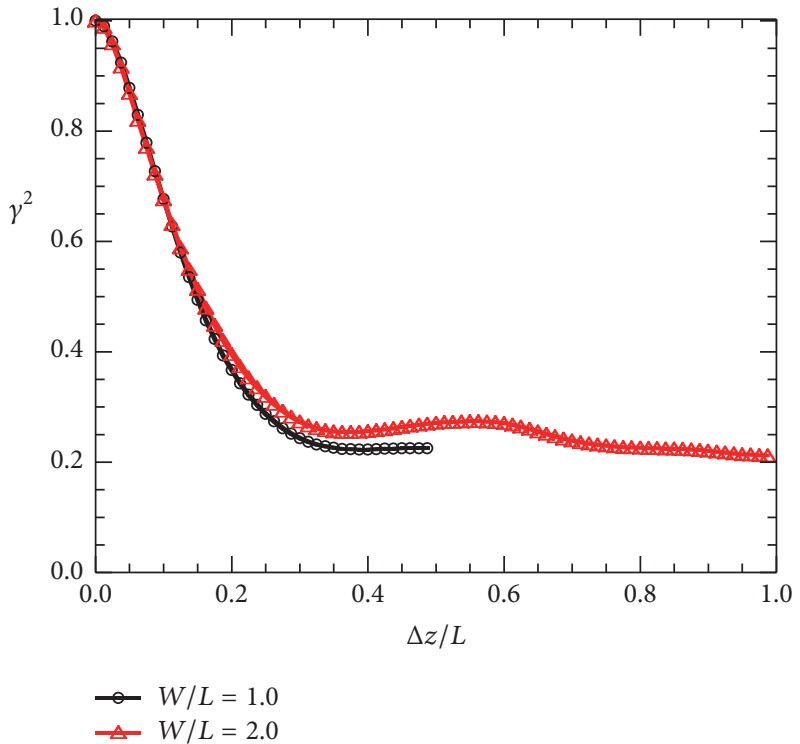

Figure 9: Predicted coherence of velocity, $v / U_{\infty}$, at fundamental frequency in spanwise direction $(x / L=0.5$ and $y=0)$ for cavity flow $(D / L=0.5)$ with original and wider computational domains of $W / L$ $=1.0$ and 2.0 .

5.2. Sound Pressure Level. Figures 10(a) and 10(b) show the predicted and measured sound pressure spectra at the points of the far field $(x / L=6.75$ and $y / L=23.5)$ for $D / L=0.5$ and 2.5 , respectively. The measured level was affected by the background noise of the wind tunnel in the range of St $<0.5$. The bars in the figures present how the results with the same 


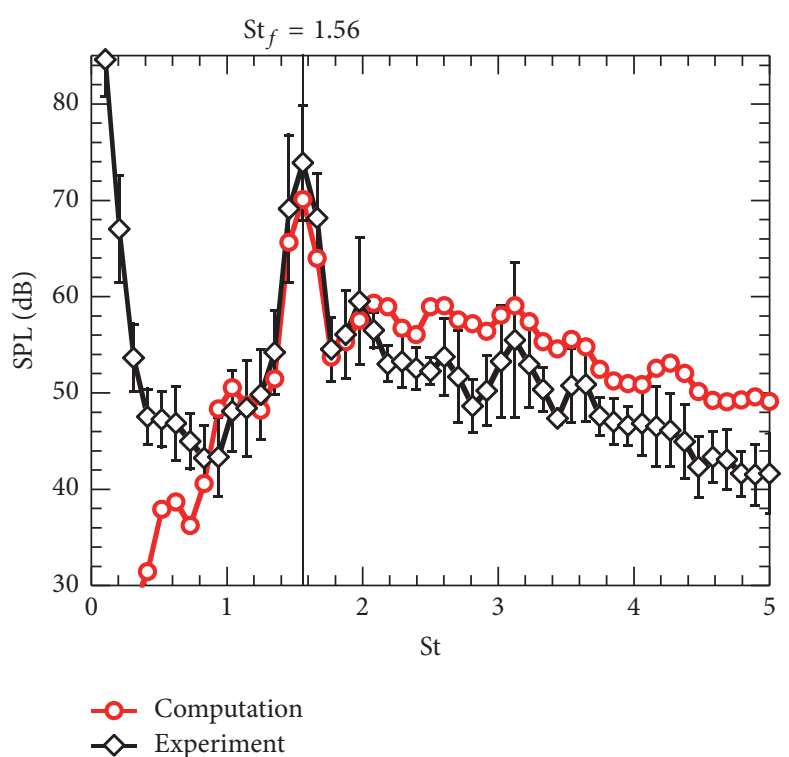

(a)

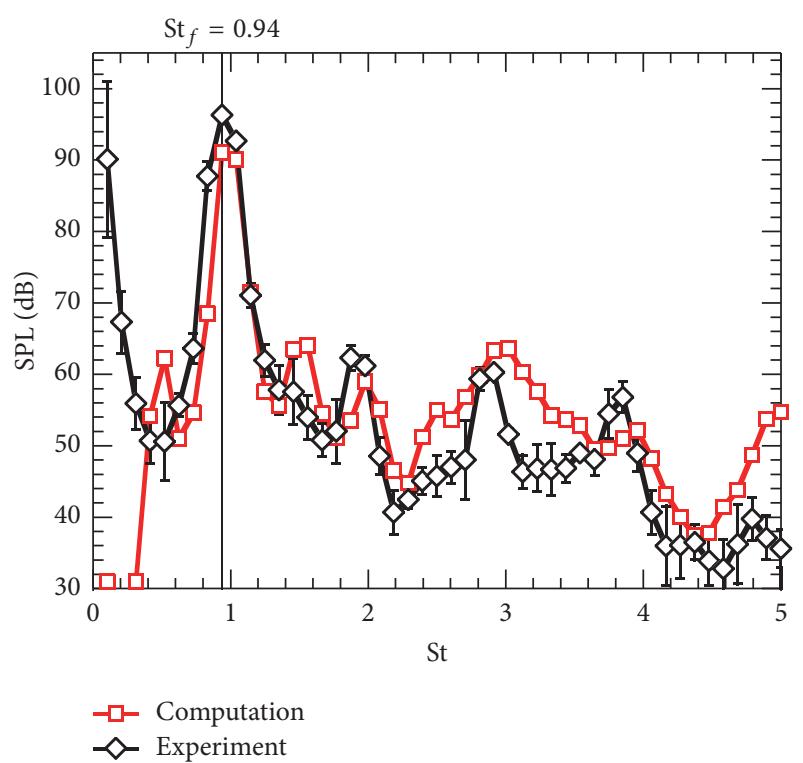

(b)

Figure 10: Comparison of predicted and measured sound pressure spectra $(x / L=6.75$ and $y / L=23.5)$. (a) Cavity flow without acoustic resonance $(D / L=0.5)$. (b) Cavity flow with acoustic resonance $(D / L=2.5)$.

averaging number of 20 as that of simulation vary around the measured value with the long averaging number of 9400 .

The predicted spectra in both cavity flows with $D / L=0.5$ and 2.5 are in good agreement with those measured. Also, the fundamental frequency for the cavity flow with $D / L=2.5$ and $\mathrm{St}_{f}=0.94$ is corresponding to the Rossiter mode of $n=2$ [1], which is the streamwise number of vortices in the shear layer, while that for $D / L=0.5$ and $\mathrm{St}_{f}=1.56$ is corresponding to $n=3$. The present peak also agrees with the resonant frequency of one-quarter wavelength mode in the direction of cavity depth, $\mathrm{St}_{\text {res }}=0.84$ and 0.98 , which are estimated by using the semiempirical formula by East [3] and by general open correction of a circular closed pipe of $8(D / 2) / 3 \pi$ [29]. The slight difference with the resonant frequency estimated by East [3] is possibly due to the conditions of the incoming boundary layer, where the cavity was placed in a turbulent boundary layer in the experiments by East.

To confirm the occurrence of the acoustic resonance in the cavity flow with $D / L=2.5$, the phase of the pressure fluctuations in the cavity was investigated. Figure 11 shows the phase differences in the pressure fluctuations, $C_{p} \equiv$ $p / 0.5 \rho_{\infty} U_{\infty}{ }^{2}$, at the fundamental frequency vertically along $x / L=0.5$. The reference point for these phase differences is at the bottom of the cavity $(y / L=-2.5)$. The phase of pressure fluctuations in the cavity is constant in the cavity $(y / L<$ $-0.1)$; that is, the standing waves due to acoustic resonance are generated in the cavity. These results indicate that acoustic resonance can be captured in the present computation.

To clarify the dependency of the predicted results on computational grid, the computations with two different meshes were also performed for cavity flow of $D / L=$ 0.5 without freestream turbulence $(\mathrm{Tu}=0.0 \%)$. One mesh consists of finer mesh of double grid points in the normal

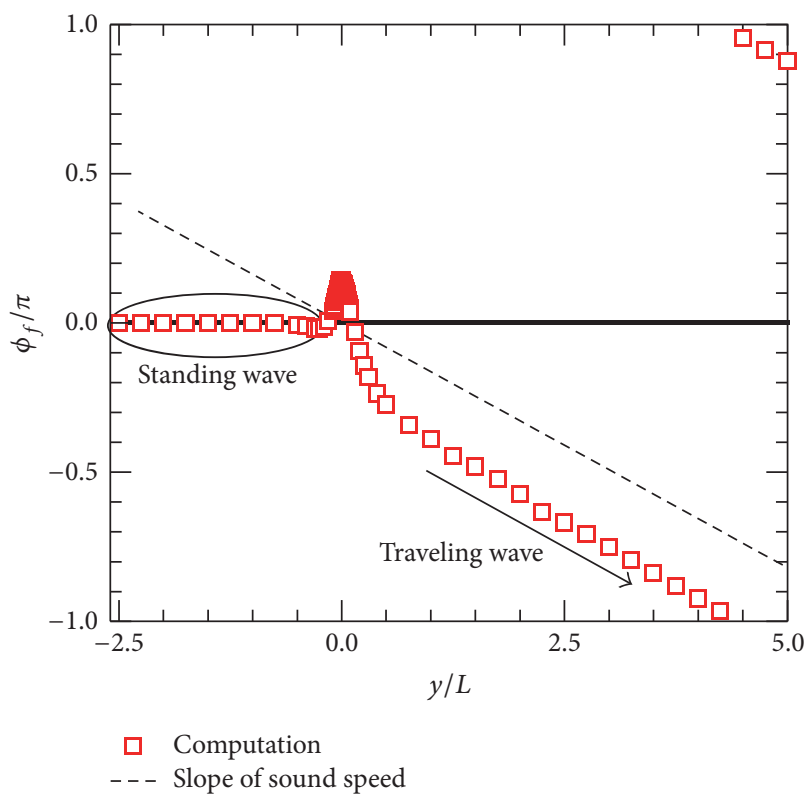

FIGURE 11: Predicted phase differences of pressure fluctuations, $C_{p} \equiv$ $p / 0.5 \rho_{\infty} U_{\infty}{ }^{2}$, at fundamental frequency vertically along $x / L=0.5$ for cavity flow with acoustic resonance $(D / L=2.5)$.

direction in the cavity, where the normal stretch of grid resolution is lower than that of original mesh. The other one is twice as wide as the present mesh as mentioned in Section 5.1.

Figure 12 shows the predicted sound pressure spectra with these meshes. As shown in this figure, the tonal sound is predicted at the same fundamental frequency of $\mathrm{St}_{f}=1.56$ and 


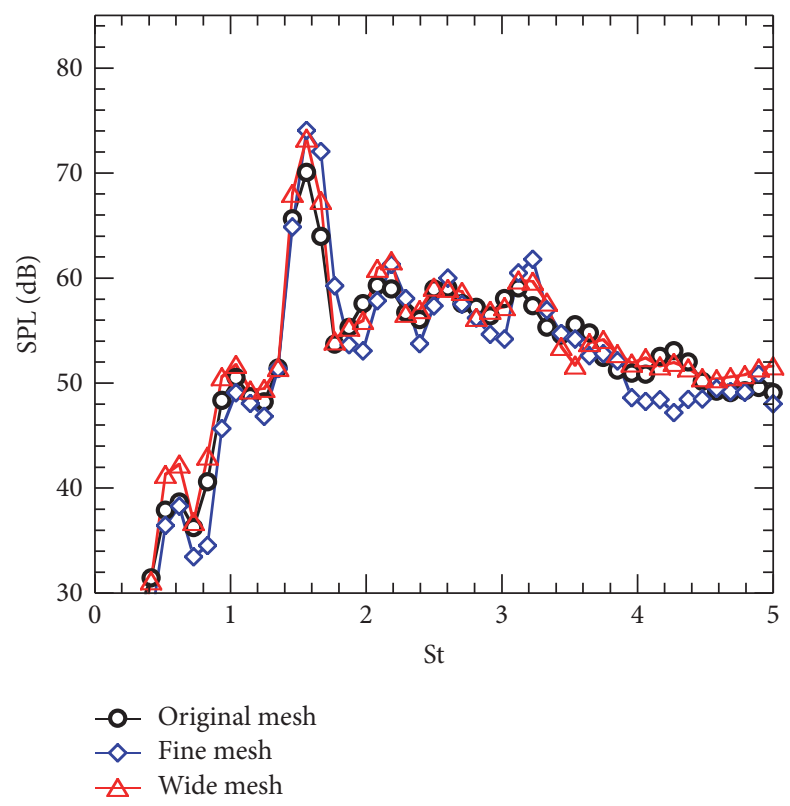

FIGURE 12: Comparison of predicted sound pressure spectra $(x / L=$ 6.75 and $y / L=23.5$ ) with original, finer, and wider meshes for cavity flow without freestream turbulence $(D / L=0.5)$.

the difference of level is within $5 \mathrm{~dB}$. Therefore, it has been concluded that the dependency of the predicted results on the mesh is small.

It has been concluded along with the discussions of flow field in Section 5.1 that the present computations could adequately capture the flow and acoustic fields.

\section{Results and Discussion}

6.1. Shear Layer in Cavity Flow. Figure 13 shows the predicted mean velocity and turbulent intensity at the center of the cavity $(x / L=0.5)$ for the cavity flow with $D / L=0.5$ with and without freestream turbulence. As shown in Figure 13(a), it is clarified that the shear layer is thickening with freestream turbulence.

The momentum thickness $\delta_{m}$ at $x / L=0.5$ was computed as

$$
\delta_{m}=\int_{y_{\min }}^{y_{\max }} \frac{U(y)}{U_{\infty}}\left(1-\frac{U(y)}{U_{\infty}}\right) d y
$$

where $y_{\min } / L$ and $y_{\max } / L$ were set to be -0.2 and 0.2 . This range is the same as that in reference [30]. The momentum thickness was clarified to become thicker from $\delta_{m} / L=0.019$ $(\mathrm{Tu}=0.0 \%)$ to $0.027(\mathrm{Tu}=2.3 \%)$ by the freestream turbulence. Figure 13(b) also shows that the turbulent intensity becomes more intense by the freestream turbulence.

Figure 14 shows the predicted profiles for the cavity flow with $D / L=2.5$. As shown in this figure, the shear layer becomes thicker, where the momentum thickness was changed from $\delta_{m} / \mathrm{L}=0.041(\mathrm{Tu}=0.0 \%)$ to $0.049(\mathrm{Tu}=$ $2.3 \%$ ) by the freestream turbulence. Figure 14 shows that the peak of the turbulent intensity becomes slightly weaker by the freestream turbulence. This is because the velocity fluctuations at the fundamental frequency become weaker. The effects of the freestream turbulence on velocity spectra are discussed in the next section. Also, as shown in Figure 14, the region for intense turbulent intensity is spread in the normal direction by the freestream turbulence of $\mathrm{Tu}=2.3 \%$. As a result, the mixing in the shear layer is enhanced and the shear layer becomes thicker.

6.2. Vortical Structures. Figure 15 shows instantaneous flow fields around the cavity for $\mathrm{Tu}=0.0 \%$ and $2.3 \%$ for the cavity flow without acoustic resonance $(D / L=0.5)$. Figure 16 shows those for the cavity flow with acoustic resonance $(D / L=2.5)$.

Large-scale vortices are found for the cavity flow without freestream turbulence $(\mathrm{Tu}=0.0 \%)$ for both cavity flows with and without acoustic resonance. These vortices are shed from the upstream edge of the cavity at the fundamental frequency and contribute to the periodic acoustic radiation from the cavity.

The large-scale vortical structures, which are coherent in the spanwise direction, are dominant for $\mathrm{Tu}=0.0 \%$ particularly in the cavity flow with acoustic resonance. Also, streamwise rib structures and fine scale eddies in the shear layer can be observed for $\mathrm{Tu}=0.0 \%$ for the cavity flow without acoustic resonance. As the turbulent intensity is increased, the fine scale vortices become active regardless of acoustic resonance.

Figures 17(a) and 17(b) show the power spectra of the vertical velocity, $v / U_{\infty}$, at the center of the cavity $(x / L=0.5$ and $y=0$ ), where large-scale vortices are observed without freestream turbulence, for the cavity flow without acoustic resonance and that with acoustic resonance, respectively.

The power spectra for freestream turbulence $\mathrm{Tu}=0.9 \%$ are almost the same as that for the cavity flow without freestream turbulence $(\mathrm{Tu}=0.0 \%)$ in both cases with and without acoustic resonance. The level of velocity fluctuations at the fundamental frequency becomes significantly lower for $\mathrm{Tu}=2.3 \%$. This level corresponds to the power of velocity fluctuations due to the large-scale vortical structures, which became the sound source of the cavity tone.

These spectra are also compared with the curve for $-5 / 3$ power. All the slopes of the power spectra of velocity fluctuations with and without freestream turbulence in the range of $S t=1-5$ are along the curve of $-5 / 3$ power for the cavity flow without acoustic resonance. Also, the level in that range is approximately independent of freestream turbulence.

As shown in Figure 17(b), for the cavity flow with acoustic resonance, the slope of the spectrum becomes close to the curve for $-5 / 3$ power by adding freestream turbulence. Figures 18(a) and 18(b) show the power spectra near the downstream edge of the cavity $(x / L=0.9$ and $y=0)$ for the cavity flow without acoustic resonance and that with acoustic resonance, respectively. Figure 18(b) shows that the slope of the power is clearly along the curve of $-5 / 3$ power in the range of St $=1-5$ particularly for $\mathrm{Tu}=2.3 \%$ for the cavity flow with acoustic resonance. Also, the power at high frequencies of St $\geq 3$ becomes more intense with the freestream turbulence of $\mathrm{Tu}=2.3 \%$.

The above-mentioned results indicate that turbulent mixing in the shear layer is enhanced by freestream turbulence, 


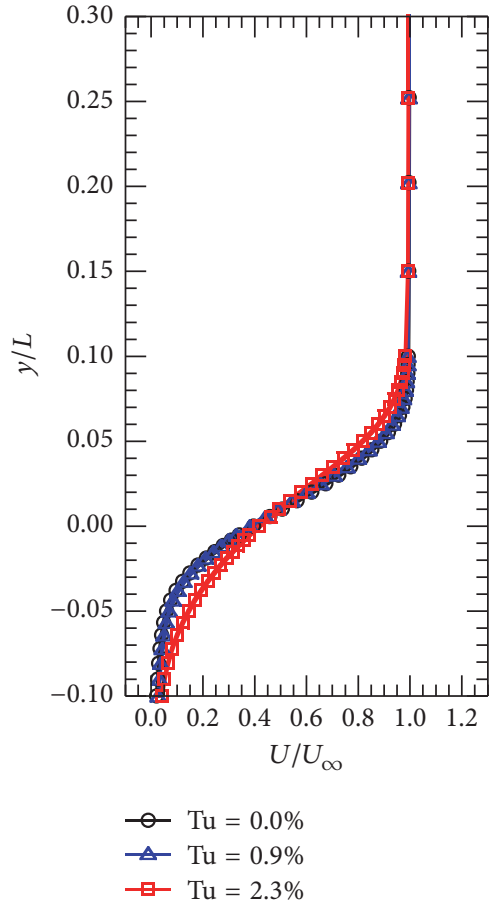

(a)

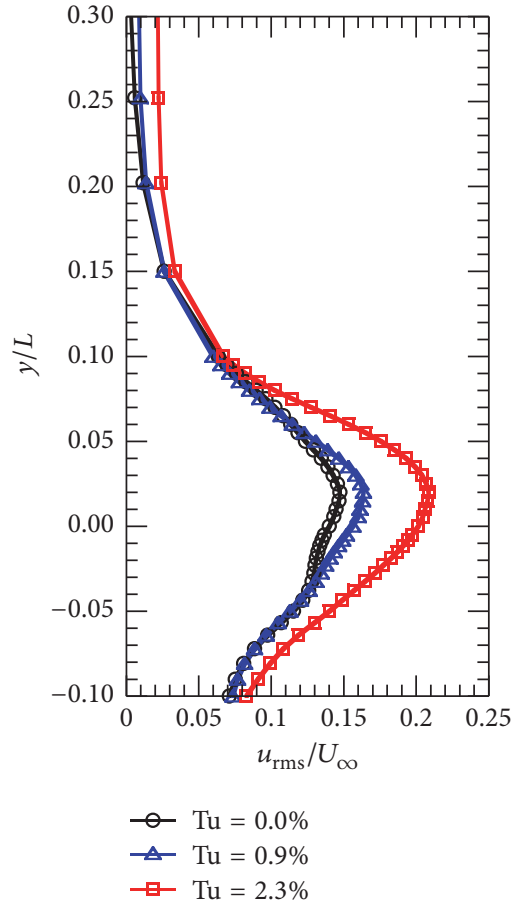

(b)

FIGURE 13: Predicted profiles of mean velocity (a) and turbulent intensity (b) for the cavity flow with $D / L=0.5$.

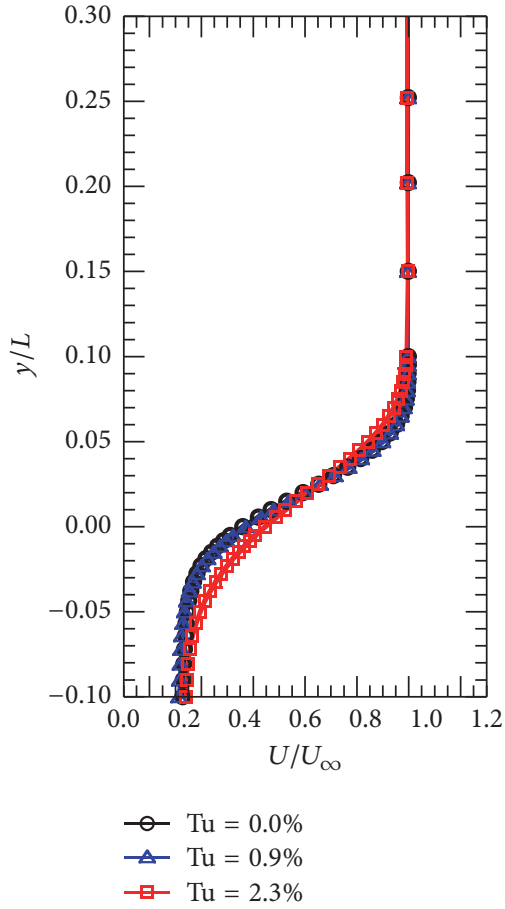

(a)

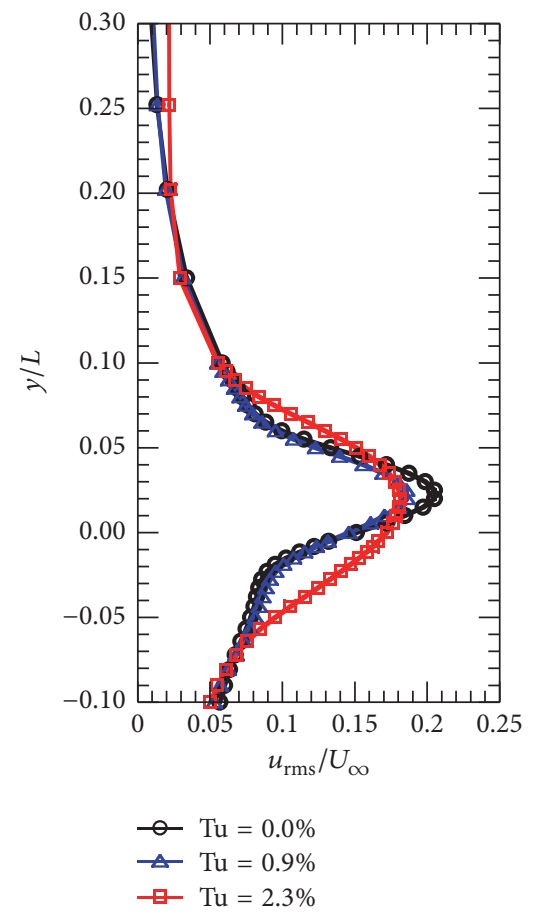

(b)

FIGURE 14: Predicted profiles of mean velocity (a) and turbulent intensity (b) for the cavity flow with $D / L=2.5$. 


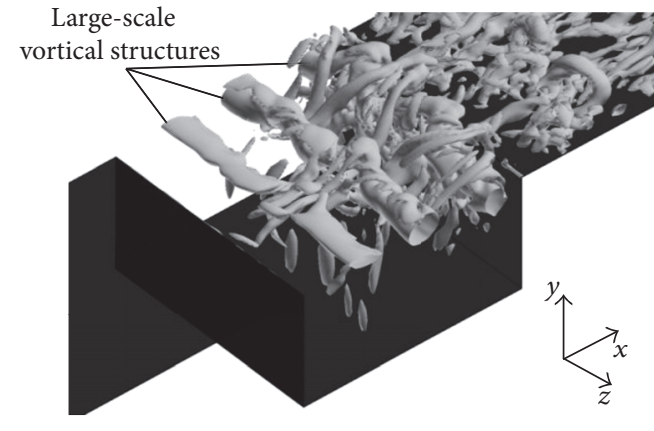

(a)

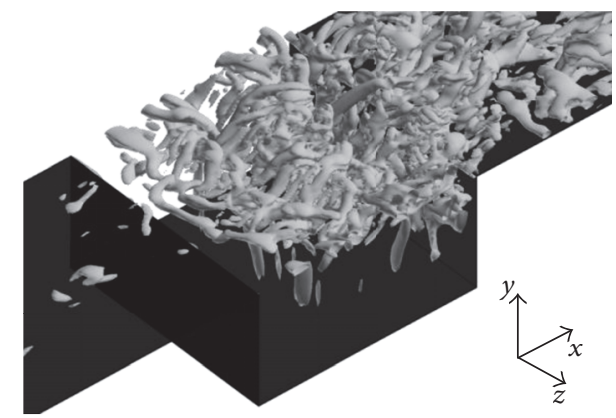

(b)

FIGURE 15: Instantaneous isosurfaces of second invariant of velocity tensor gradient $\left(q /\left(U_{\infty} / L\right)^{2}=20\right)$ for cavity flows without acoustic resonance $(D / L=0.5)$. (a) Cavity flow without freestream turbulence $(\mathrm{Tu}=0.0 \%)$. (b) Cavity flow with freestream turbulence, Tu $=2.3 \%$.

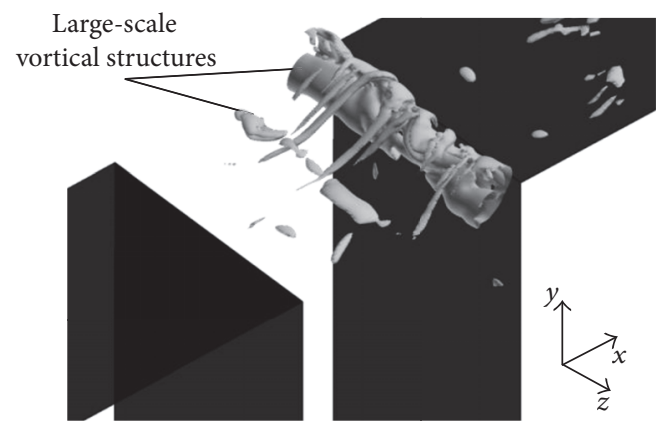

(a)

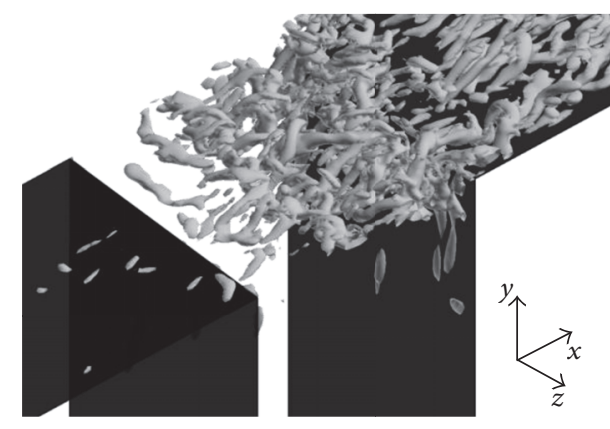

(b)

FIGURE 16: Instantaneous isosurfaces of second invariant of velocity tensor gradient $\left(q /\left(U_{\infty} / L\right)^{2}=20\right)$ for cavity flows with acoustic resonance $(D / L=2.5)$. (a) Cavity flow without freestream turbulence $(\mathrm{Tu}=0.0 \%)$. (b) Cavity flow with freestream turbulence, $\mathrm{Tu}=2.3 \%$.

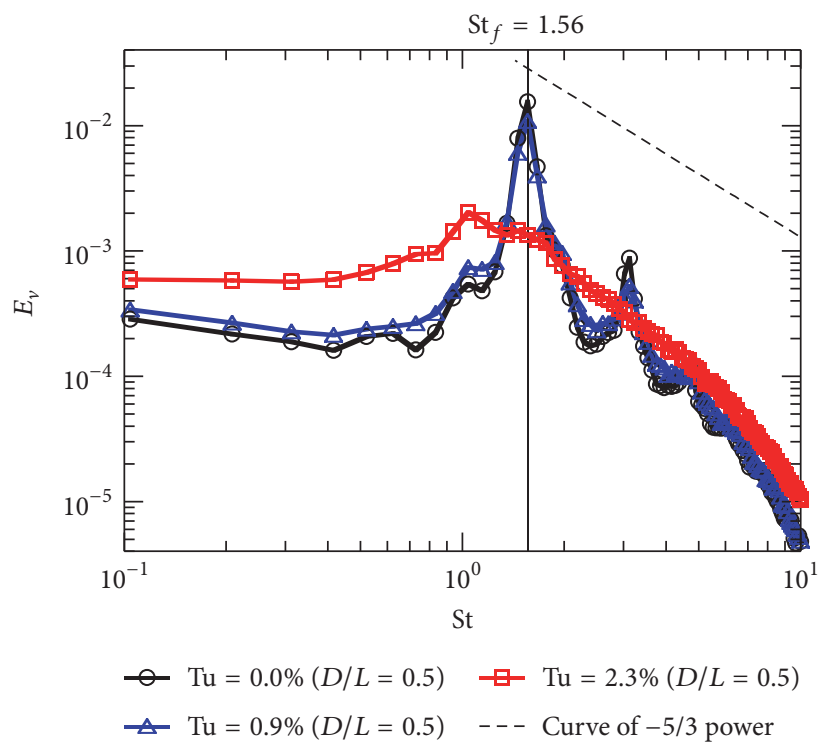

(a)

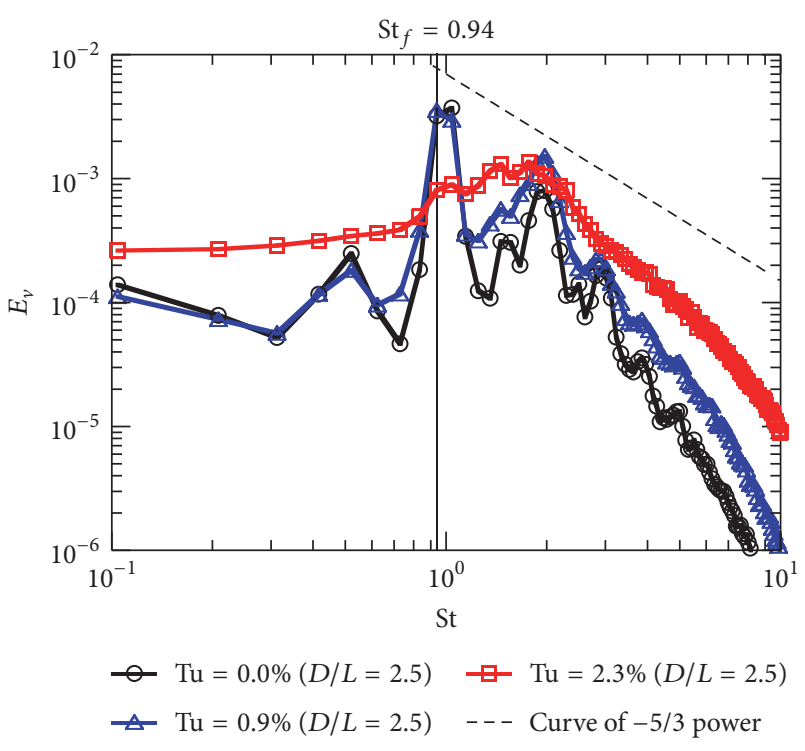

(b)

FIGURE 17: Power spectra of velocity $v / U_{\infty}(x / L=0.5$ and $y=0)$. (a) Cavity flow without acoustic resonance $(D / L=0.5)$. (b) Cavity flow with acoustic resonance $(D / L=2.5)$. 


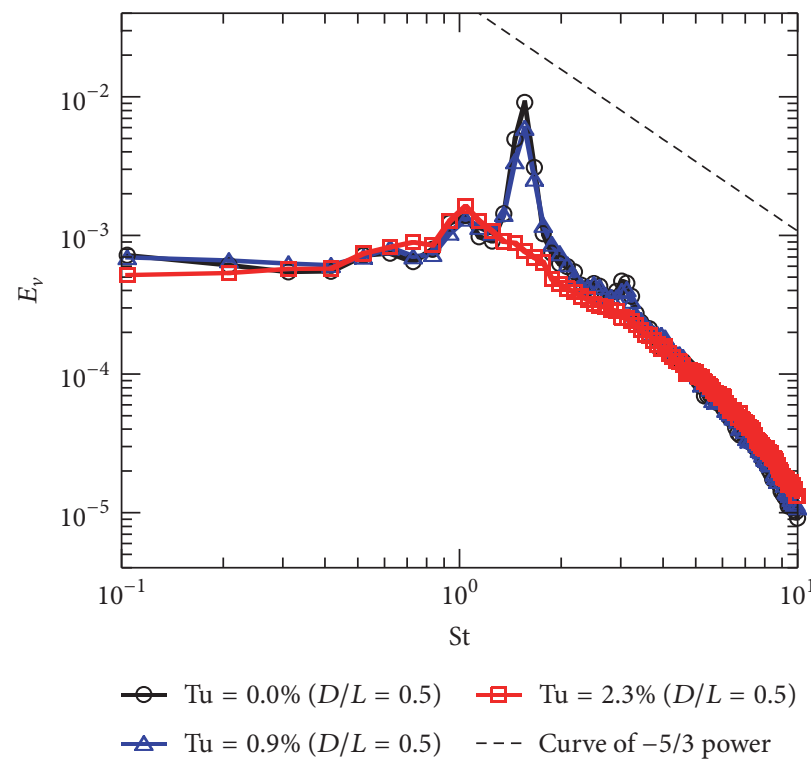

(a)

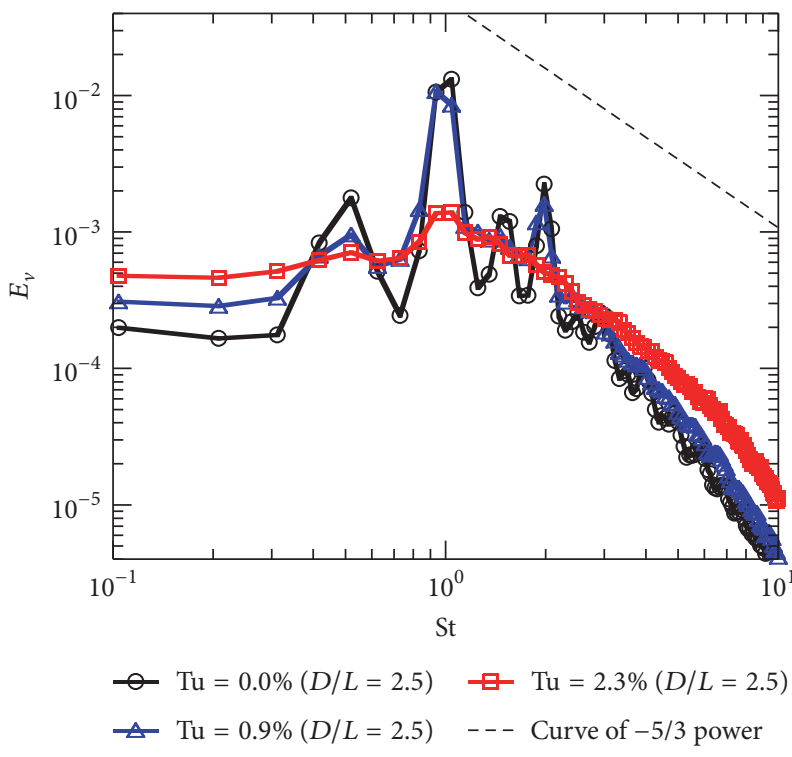

(b)

FIGURE 18: Power spectra of velocity $v / U_{\infty}(x / L=0.9$ and $y=0)$. (a) Cavity flow without acoustic resonance $(D / L=0.5)$. (b) Cavity flow with acoustic resonance $(D / L=2.5)$.

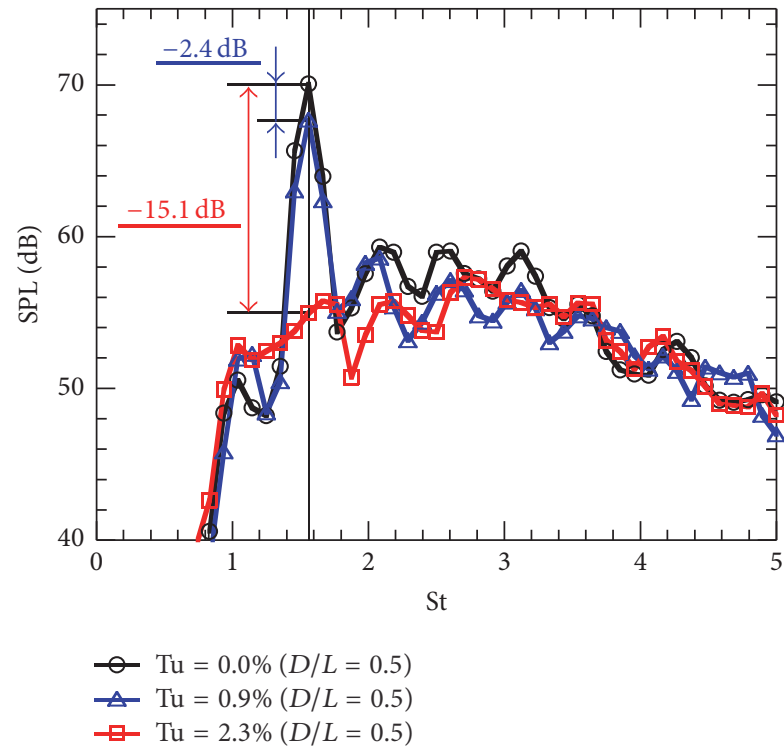

(a)

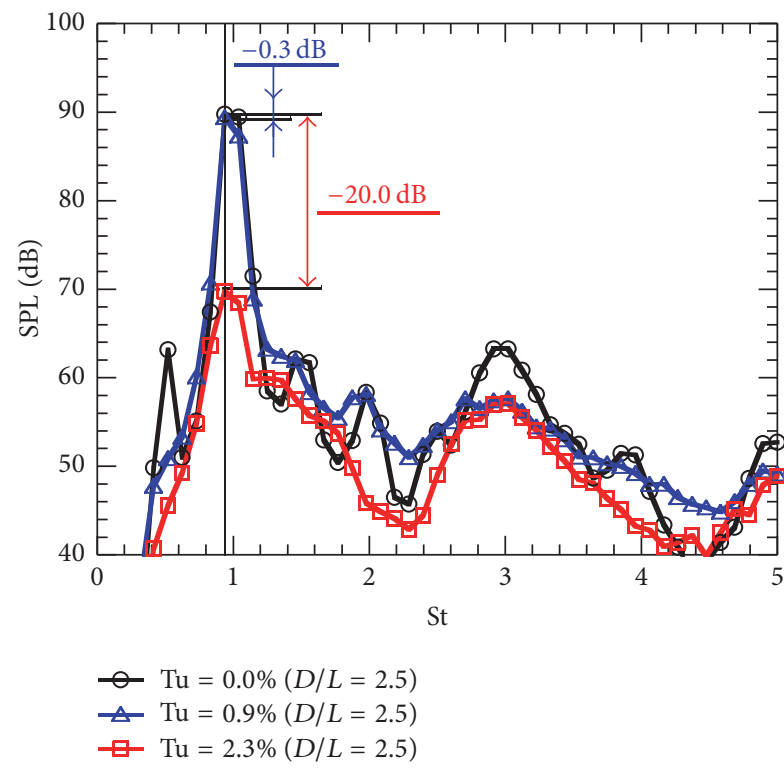

(b)

FiguRE 19: Sound pressure spectra $(x / L=6.75$ and $y / L=23.5)$ and difference in tonal sound pressure levels due to effects of freestream turbulence. (a) Cavity flow without acoustic resonance $(D / L=0.5)$. (b) Cavity flow with acoustic resonance $(D / L=2.5)$.

and the turbulent transition in the shear layer occurs due to freestream turbulence.

6.3. Effects of Freestream Turbulence on Cavity Tone. Figure 19 shows sound pressure spectra in the acoustic far field $(x / L=$ 6.75 and $y / L=23.5)$ for cavity flows with and without acoustic resonance. The differences in the tonal sound pressure levels at the fundamental frequency, $\Delta \mathrm{SPL}$, with reference to that for the cavity flow without freestream turbulence are also presented.

The $\Delta$ SPL is $-2.4 \mathrm{~dB}$ and $-15.1 \mathrm{~dB}$ for $\mathrm{Tu}=0.9$ and $2.3 \%$, respectively, for the cavity flow without acoustic resonance. The $\triangle$ SPL is $-0.3 \mathrm{~dB}$ and $-20.0 \mathrm{~dB}$ for $\mathrm{Tu}=0.9$ and $2.3 \%$, respectively, for the cavity flow with acoustic resonance.

The effects of freestream turbulence on the cavity tone are less than $2.5 \mathrm{~dB}$ for both cavity flows with and without acoustic resonance for freestream turbulence of $\mathrm{Tu}=0.9 \%$. 


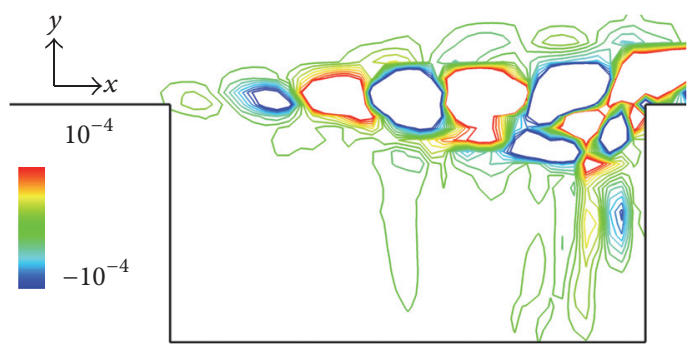

(a)

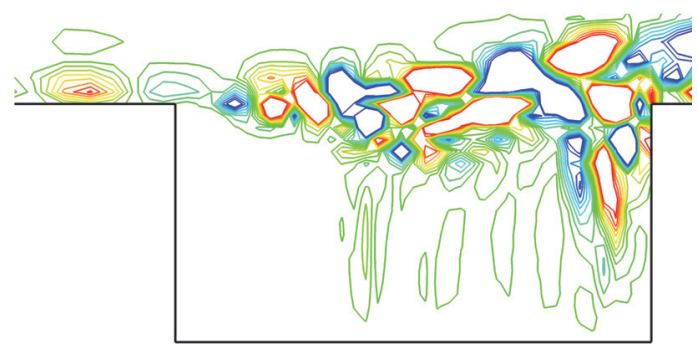

(b)

Figure 20: Contours of real part of power of Lighthill's stress tensor at fundamental frequency, $\partial^{2} T_{i j} / \partial x_{i} \partial x_{j} /\left(\rho_{\infty} U_{\infty}{ }^{2} / L^{2}\right)$, around cavity for cavity flows without acoustic resonance $(D / L=0.5)$. (a) Cavity flow without freestream turbulence $(\mathrm{Tu}=0.0 \%)$. (b) Cavity flow with freestream turbulence, $\mathrm{Tu}=2.3 \%$.

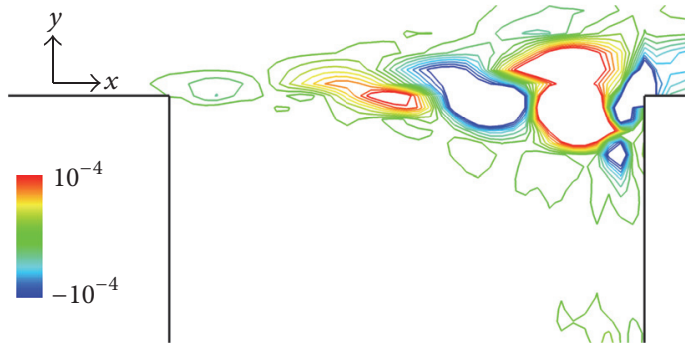

(a)

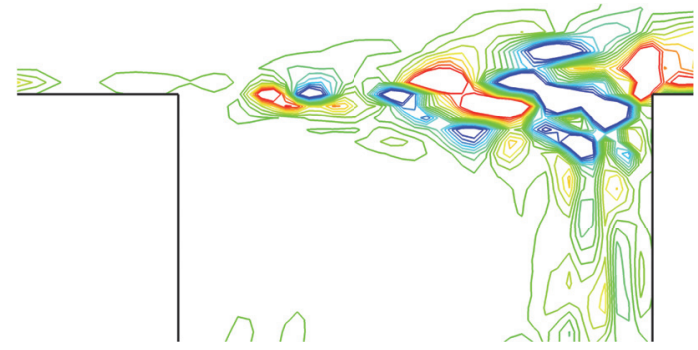

(b)

FIGURE 21: Contours of real part of power of Lighthill's stress tensor at fundamental frequency, $\partial^{2} T_{i j} / \partial x_{i} \partial x_{j} /\left(\rho_{\infty} U_{\infty}{ }^{2} / L^{2}\right)$, around cavity for cavity flows with acoustic resonance $(D / L=2.5)$. (a) Cavity flow without freestream turbulence $(\mathrm{Tu}=0.0 \%)$. (b) Cavity flow with freestream turbulence, $\mathrm{Tu}=2.3 \%$.

Tonal sound generated from the cavity flow with acoustic resonance is greatly affected by freestream turbulence for $\mathrm{Tu}=2.3 \%$.

The weakening of the intensity and lowering of the spanwise coherence of the sound source are considered to be responsible for the reduced cavity tone. The following sections discuss the effects of the freestream turbulence on the intensity and spanwise coherence of the sound source.

6.4. Intensity of Sound Source. Aerodynamic sound is related to the intensity of fluctuating pressures/velocities and their structures (coherent motion) according to acoustic analogies $[11,13,22]$. In addition, the velocity fluctuations near the downstream edge of the cavity contribute to the sound pressure level of the cavity tone since the cavity tone is generated by impinging large-scale vortical structures at the downstream edge of the cavity [17].

The Lighthill's equations [13] are

$$
\begin{aligned}
\frac{\partial^{2} \rho}{\partial t^{2}}-c^{2} \frac{\partial^{2} \rho}{\partial x_{i}^{2}} & =\frac{\partial^{2} T_{i j}}{\partial x_{i} \partial x_{j}}, \\
T_{i j} & \approx \rho u_{i} u_{j},
\end{aligned}
$$

where tensor $T_{i j}$ is Lighthill's stress tensor. This tensor is approximately equal to the Reynolds stress term at the low Mach number [31] as shown in (6). In the present section, the intensity of the sound source was estimated by using the
Reynolds stress term of Lighthill's stress tensor [13] at the fundamental frequency near the downstream edge $(0.75 \leq$ $x / L \leq 1.0)$. The range of $0.75 \leq x / L \leq 1.0$ is approximately equal to the scale of the large-scale vortical structures.

Figures 20 and 21 show the intensity of the abovementioned sound source (the Reynolds stress term of Lighthill's stress tensor) at the fundamental frequency, around the cavity. Also, Figure 22 shows the power level of the sound source at the fundamental frequency, $P_{f}$, along $y=0$ from the upstream edge to the downstream edge. As shown in the figure, the acoustic source is weakened by the freestream turbulence.

Moreover, the sound reduction level of the cavity tone due to the weakening of the intensity of the sound source, $\Delta \mathrm{SPL}_{i}$, was calculated by the integration of the power of source, $P_{f}$, in the range of $0.75 \leq x / L \leq 1.0$. The equation of $\Delta \mathrm{SPL}_{i}$ is as follows:

$$
\Delta \mathrm{SPL}_{i}=10 \log _{10}\left[\int_{0.75 L}^{L}\left(\frac{P_{f}}{P_{f 0}}\right) d x\right],
$$

where the subscript " 0 " means the condition of the cavity flow without freestream turbulence $(\mathrm{Tu}=0.0 \%)$.

Table 2 shows the values of $\triangle \mathrm{SPL}_{i}$ in each condition. The value of $\Delta \mathrm{SPL}_{i}$ is $-8.5 \mathrm{~dB}$ and $-8.2 \mathrm{~dB}$ for $\mathrm{Tu}=2.3 \%$ with and without acoustic resonance, respectively, which represent greater effects than those for $\mathrm{Tu}=0.9 \%(-0.3 \mathrm{~dB}$ and $0.8 \mathrm{~dB})$. Also, this means that the cavity tone is greatly reduced by weakening of the sound source for $\mathrm{Tu}=2.3 \%$. 


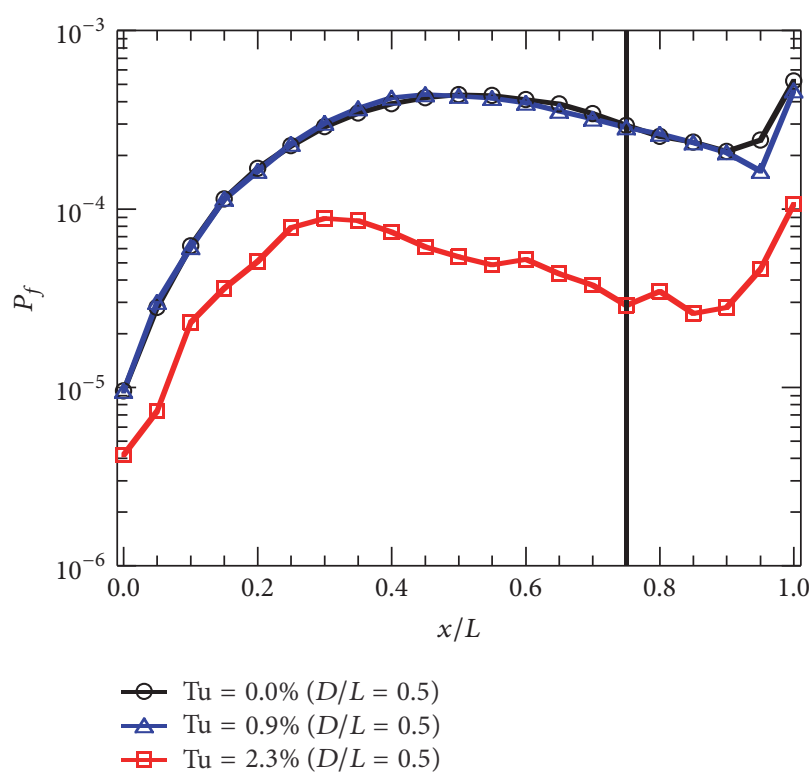

(a)

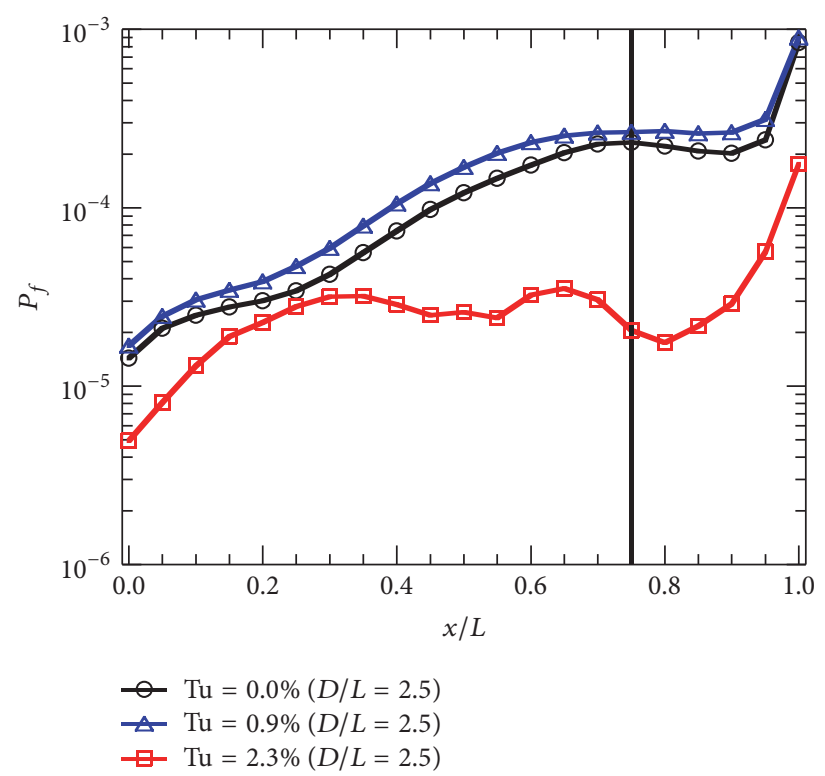

(b)

FIGURE 22: Power level of Lighthill's stress tensor at the fundamental frequency, $P_{f}$, along $y=0$. (a) Cavity flow without acoustic resonance $(D / L=0.5)$. (b) Cavity flow with acoustic resonance $(D / L=2.5)$.

TABLE 2: Contributions of intensity and spanwise coherence of sound source to the sound pressure level.

\begin{tabular}{|c|c|c|c|c|c|c|}
\hline$D / L$ & $\mathrm{Tu}[\%]$ & $L_{c} / L$ & $\Delta \mathrm{SPL}[\mathrm{dB}]$ & $\Delta \mathrm{SPL}_{i}[\mathrm{~dB}]$ & $\Delta \mathrm{SPL}_{c}[\mathrm{~dB}]$ & $\Delta \mathrm{SPL}_{\text {total }}[\mathrm{dB}]$ \\
\hline \multirow{3}{*}{0.5} & 0 & 0.22 & - & - & - & - \\
\hline & 0.9 & 0.12 & -2.4 & -0.3 & -2.7 & -3.0 \\
\hline & 2.3 & 0.04 & -15.1 & -8.5 & -4.2 & -12.7 \\
\hline \multirow{3}{*}{2.5} & 0 & 6.40 & - & - & - & - \\
\hline & 0.9 & 5.76 & -0.3 & 0.8 & -0.5 & 0.3 \\
\hline & 2.3 & 0.05 & -20.0 & -8.2 & -17.1 & -25.3 \\
\hline
\end{tabular}

6.5. Spanwise Coherence of Sound Source. Two-dimensionality in the spanwise direction of large-scale vortical structures as shown in Figures 15 and 16 is quantitatively discussed. In the present section, the effects of freestream turbulence on the spanwise coherence of the sound source are discussed by computing the coherence of the vertical velocity, $v / U_{\infty}$, at the fundamental frequency at the center of the cavity $(x / L=$ 0.5 and $y=0$ ), which is shown in Figure 23.

As shown in Figure 23, the coherence is decreased by the freestream turbulence for both cases with and without acoustic resonance. The equivalent coherent length, $L_{c} / L$, which was discussed in Section 4.4, is $0.22,0.12$, and 0.04 for the flow with freestream turbulence of $\mathrm{Tu}=0.0,0.9$, and $2.3 \%$, respectively, in the cavity flow without acoustic resonance, whereas it is $6.40,5.76$, and 0.05 for $\mathrm{Tu}=0.0,0.9$, and $2.3 \%$ for the cavity flow with acoustic resonance.

Equivalent coherent length for the cavity flow with acoustic resonance without freestream turbulence, $L_{c} / L=6.40$, is greater than that for the flow without acoustic resonance $\left(L_{c} / L=0.22\right)$. This means that the two-dimensionality of the large-scale vortices related with cavity tone becomes higher due to the acoustic resonance. As shown in Figures 15 and 16, three-dimensional fine scale vortices are less active for cavity flow with acoustic resonance.

In Section 4.4, the method for correction of SPL for the difference between the computational and experimental spanwise extent is discussed. With a similar method, the effects of lowering spanwise coherence of the sound source on the cavity tone, $\Delta \mathrm{SPL}_{c}$, are estimated as follows:

$$
\begin{aligned}
\mathrm{SPL}_{c} & =10 \log _{10}\left(\frac{W_{e}}{\lambda_{v}}\right) \quad\left(L_{c} \leq \lambda_{v}\right), \\
\mathrm{SPL}_{c} & =20 \log _{10}\left(\frac{L_{c}}{\lambda_{v}}\right)+10 \log _{10}\left(\frac{W_{e}}{L_{c}}\right) \\
& \left(\lambda_{v}<L_{c} \leq W_{e}\right), \\
\mathrm{SPL}_{c} & =20 \log _{10}\left(\frac{W_{e}}{\lambda_{v}}\right) \quad\left(W_{e}<L_{c}\right), \\
\Delta \mathrm{SPL}_{c} & =\mathrm{SPL}_{c}-\mathrm{SPL}_{c 0},
\end{aligned}
$$

where $\lambda_{v}$ is the streamwise scale of vortices related to cavity tone and estimated to be approximately $L /(4 n)$ by the distance of the streamwise phase difference of velocity fluctuations of $\Delta \phi<\pi / 2$ in the shear layer. 


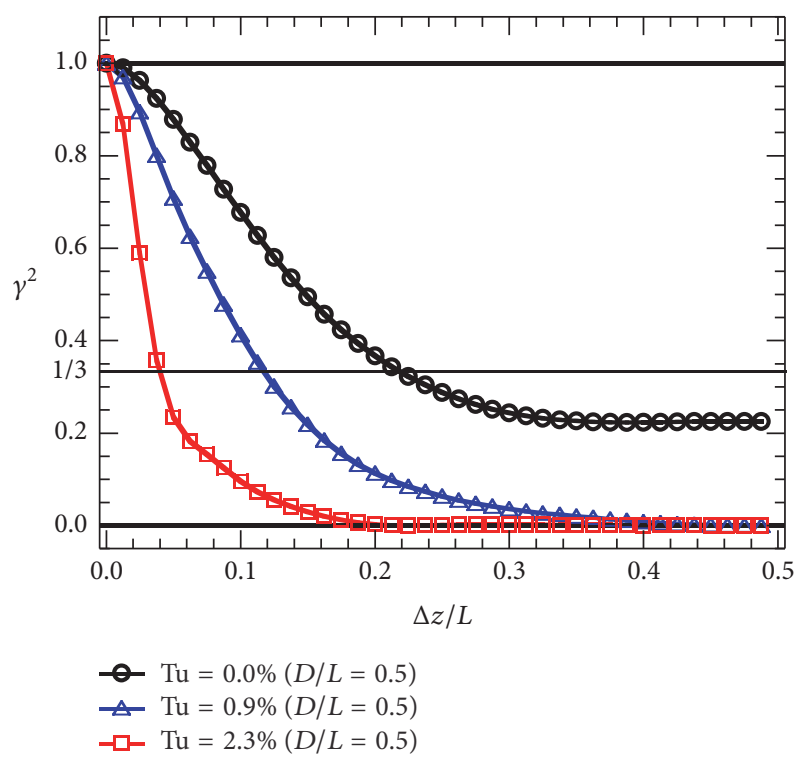

(a)

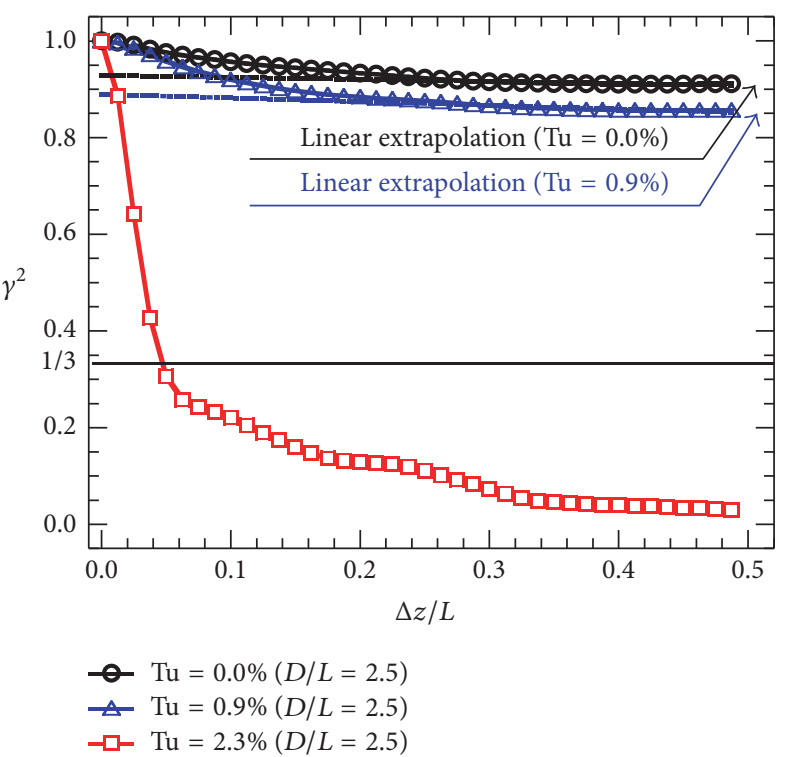

(b)

Figure 23: Coherence of velocity $v / U_{\infty}$ at fundamental frequency in spanwise direction $(x / L=0.5$ and $y=0)$. (a) Cavity flow without acoustic resonance $(D / L=0.5)$. (b) Cavity flow with acoustic resonance $(D / L=2.5)$.

Table 2 also shows the value of $\Delta \mathrm{SPL}_{c}$ for each case. It is $-0.6 \mathrm{~dB}$ and $-4.2 \mathrm{~dB}$ for $\mathrm{Tu}=0.9$ and $2.3 \%$ in the cavity flow without acoustic resonance, while it is $-0.5 \mathrm{~dB}$ and $-17.1 \mathrm{~dB}$ for $\mathrm{Tu}=0.9$ and $2.3 \%$ for the cavity flow with acoustic resonance. These results present that the effects of the freestream turbulence in the cavity flow with acoustic resonance were greater than those in the flow without acoustic resonance. This is possibly because the turbulent transition occurs in the free shear layer of the flow with acoustic resonance while the shear layer is turbulent even without freestream turbulence in the flow without acoustic resonance.

6.6. Contributions of Intensity and Spanwise Coherence of Sound Source. Both the intensity and spanwise coherence of the sound source affect variation of the sound pressure level by freestream turbulence, as was previously mentioned. The contributions of these two factors are discussed in the present section. Table 2 and Figure 24 help to explain the contributions of various factors to the cavity tone. The total difference in the cavity tone due to contributions by these factors, $\Delta \mathrm{SPL}_{\text {total }}$, is calculated as

$$
\Delta \mathrm{SPL}_{\text {total }}=\Delta \mathrm{SPL}_{i}+\Delta \mathrm{SPL}_{c}
$$

Figure 24 shows that the reduction level estimated with all factors, $\Delta \mathrm{SPL}_{\text {total }}$, is comparable to the predicted one from the direct simulation, $\triangle \mathrm{SPL}$, for each case. Also, we found qualitative agreement such that the effects of freestream turbulence on the cavity tone were greater in the cavity flow with acoustic resonance than that without acoustic resonance. This is because the reduced sound pressure level due to the reduction of the coherence is greater for the cavity flow with acoustic resonance, where the turbulent transition occurs. Also, this agreement shows that the present method for estimating the effects of the intensity and coherence of the sound source on the cavity tone is reasonable.

\section{Conclusions}

Direct simulations of flow and acoustic field were carried out for cavity flows with and without acoustic resonance $(D / L=$ 0.5 and 2.5) under various freestream turbulent conditions to clarify the effects of freestream turbulence on the cavity tone. The freestream Mach number was $M=0.09$ and the Reynolds number based on the cavity length was $\operatorname{Re}_{L}=$ $4.0 \times 10^{4}$. The incoming boundary layer was laminar with momentum thickness $\theta / L=0.0071-0.0074$. The effects of turbulent intensities of $\mathrm{Tu}=0.0-2.3 \%$ on flow and acoustic fields were investigated.

The sound source was estimated by using the flow field based on Lighthill's acoustic analogy. Moreover, the reduced cavity tone was decomposed into the effects of the intensity and spanwise coherence of the sound source to enable the reduction mechanism of the cavity tone to be understood. The total reduction level by summing these contributions by the intensity and coherence was clarified to be comparable to that predicted by direct simulations. Also, these analytical results can explain well the effects of the acoustic resonance and freestream turbulence on the reduced level, which were predicted from direct simulations. Thus, this indicates that the method for estimating the effects of the intensity and coherence of the sound source on the cavity tone in the present study is reasonable.

The effects of freestream turbulence on the cavity tone in a cavity flow with acoustic resonance, $-20.0 \mathrm{~dB}$, were greater than those in a cavity flow without acoustic resonance, $-15.1 \mathrm{~dB}$, for a cavity flow with freestream turbulence of $\mathrm{Tu}=$ 


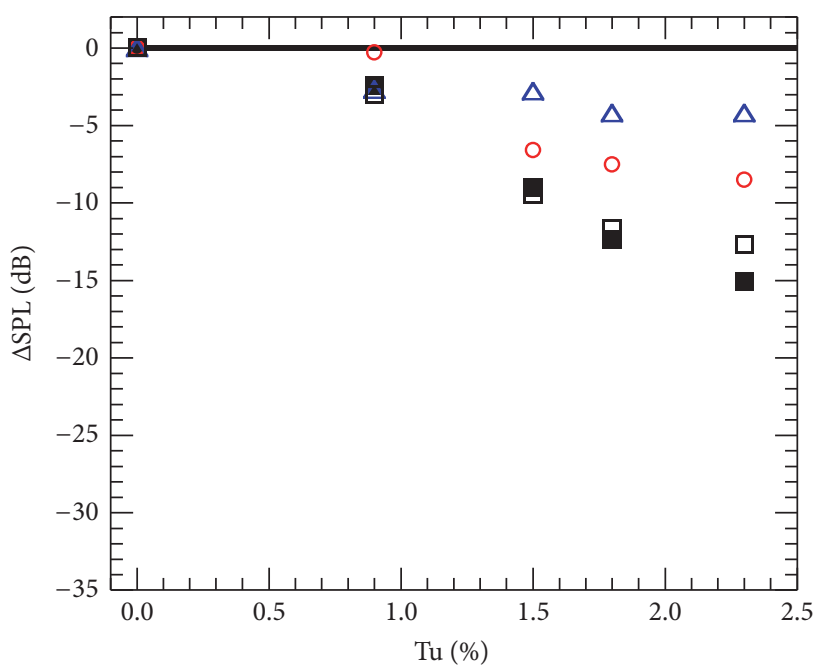

$\triangle \mathrm{SPL}$ $\circ \Delta \mathrm{SPL}_{i}$

$\Delta \Delta \mathrm{SPL}_{c}$

$\square \Delta \mathrm{SPL}_{\text {total }}$

(a)

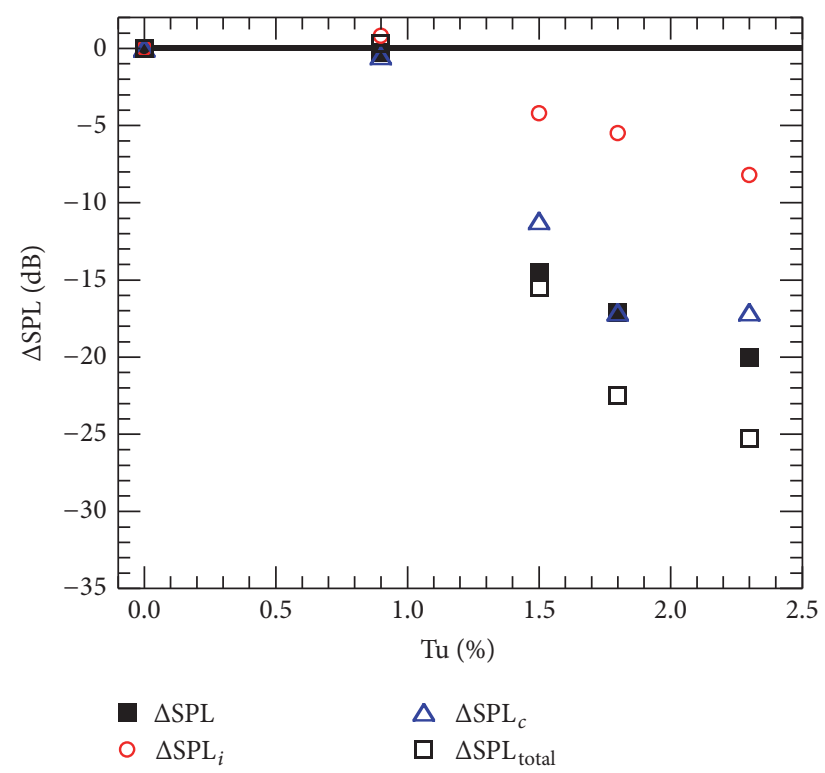

(b)

FIGURE 24: Effects of intensity and spanwise coherence of sound source on variation of cavity tone. (a) Cavity flow without acoustic resonance $(D / L=0.5)$. (b) Cavity flow with acoustic resonance $(D / L=2.5)$.

$2.3 \%$. This was caused by the difference in the flow field for the cavity flow without freestream turbulence $\mathrm{Tu}=0.0 \%$. That is, the large-scale vortical structures in the cavity flow with acoustic resonance were extremely coherent in the spanwise direction due to acoustic resonance, whereas they were less coherent even for $\mathrm{Tu}=0.0 \%$ for the cavity flow without acoustic resonance. As a result of this difference in coherence, the cavity flow with acoustic resonance was affected more by freestream turbulence.

The analyses of the intensity and spanwise coherence of the sound source clarified that the effects of the intensity and spanwise coherence of the sound source, on the other hand, were almost the same as those without acoustic resonance. The effects of spanwise coherence of the sound source on the cavity tone were greater in the cavity flow with acoustic resonance than in that without resonance.

\section{Competing Interests}

The authors declare that there is no conflict of interests regarding the publication of this paper.

\section{Acknowledgments}

The present study was supported by JSPS KAKENHI Grant nos. 24760134 and 26820044 and through the NextGeneration Supercomputer Strategy Program made available by the Ministry of Education, Culture, Sports, Science, and Technology of Japan (MEXT).

\section{References}

[1] J. E. Rossiter, "Wind-tunnel experiments on the flow over rectangular cavities at subsonic and transonic speeds," Aeronautical Research Council Report 3438, 1964.

[2] V. Sarohia, "Experimental investigation of oscillations in flows over shallow cavities," AIAA Journal, vol. 15, no. 7, pp. 984-991, 1977.

[3] L. F. East, "Aerodynamically induced resonance in rectangular cavities," Journal of Sound and Vibration, vol. 3, no. 3, pp. 277287, 1966.

[4] G. A. Brés and T. Colonius, "Three-dimensional instabilities in compressible flow over open cavities," Journal of Fluid Mechanics, vol. 599, pp. 309-339, 2008.

[5] X. Huang and X. Zhang, "Streamwise and spanwise plasma actuators for flow-induced cavity noise control," Physics of Fluids, vol. 20, no. 3, Article ID 037101, 2008.

[6] T. Lusk, L. Cattafesta, and L. Ukeiley, "Leading edge slot blowing on an open cavity in supersonic flow," Experiments in Fluids, vol. 53, no. 1, pp. 187-199, 2012.

[7] W. S. Saric, H. L. Reed, and E. J. Kerschen, "Boundary-layer receptivity to freestream disturbances," Annual Review of Fluid Mechanics, vol. 34, no. 1, pp. 291-319, 2002.

[8] L. Brandt, P. Schlatter, and D. S. Henningson, "Transition in boundary layers subject to free-stream turbulence," Journal of Fluid Mechanics, vol. 517, pp. 167-198, 2004.

[9] T. Colonius and S. K. Lele, "Computational aeroacoustics: progress on nonlinear problems of sound generation," Progress in Aerospace Sciences, vol. 40, no. 6, pp. 345-416, 2004.

[10] J. Larsson, L. Davidson, M. Olsson, and L.-E. Eriksson, "Aeroacoustic investigation of an open cavity at low mach number," AIAA Journal, vol. 42, no. 12, pp. 2462-2473, 2004. 
[11] N. Curle, "The influence of solid boundaries upon aerodynamic sound," Proceedings of the Royal Society. London. Series A. Mathematical, Physical and Engineering Sciences, vol. 231, pp. 505-514, 1955.

[12] J. Ask and L. Davidson, "Sound generation and radiation of an open two-dimensional cavity," AIAA Journal, vol. 47, no. 6, pp. 1337-1349, 2009.

[13] M. J. Lighthill, "On sound generated aerodynamically. I. General theory," Proceedings of the Royal Society. London. Series A. Mathematical, Physical and Engineering Sciences, vol. 211, pp. 564-597, 1952.

[14] K. Terao, H. Yokoyama, Y. Ogoe, and A. Iida, "Proposition of new formula for frequency prediction based on generation mechanism of aerodynamic sound in cavity flows," Transactions of the Japan Society of Mechanical Engineers B, vol. 77, no. 779, pp. 1522-1532, 2011 (Japanese).

[15] S. K. Lele, "Compact finite difference schemes with spectral-like resolution," Journal of Computational Physics, vol. 103, no. 1, pp. 16-42, 1992.

[16] K. Matsuura and C. Kato, "Large-eddy simulation of compressible transitional flows in a low-pressure turbine cascade," AIAA Journal, vol. 45, no. 2, pp. 442-457, 2007.

[17] H. Yokoyama and C. Kato, "Fluid-acoustic interactions in self-sustained oscillations in turbulent cavity flows, I. Fluiddynamic oscillations," Physics of Fluids, vol. 21, no. 10, Article ID 105103, 2009.

[18] K. W. Thompson, "Time dependent boundary conditions for hyperbolic systems," Journal of Computational Physics, vol. 68, no. 1, pp. 1-24, 1987.

[19] T. J. Poinsot and S. K. Lele, "Boundary conditions for direct simulations of compressible viscous flows," Journal of Computational Physics, vol. 101, no. 1, pp. 104-129, 1992.

[20] J. W. Kim and D. J. Lee, "Generalized characteristic boundary conditions for computational aeroacoustics," AIAA Journal, vol. 38, no. 11, pp. 2040-2049, 2000.

[21] J. O. Hinze, Turbulence, McGraw-Hill, New York, NY, USA, 1975.

[22] J. E. F. Williams and D. L. Hawkings, "Sound generation by turbulence and surfaces in arbitrary motion," Philosophical Transactions of the Royal Society of London A: Mathematical, Physical and Engineering, vol. 264, no. 1151, pp. 321-342, 1969.

[23] A. S. Lyrintzis, "Surface integral methods in computational aeroacoustics-from the (CFD) near-field to the (Acoustic) farfield," International Journal of Aeroacoustics, vol. 2, no. 2, pp. 95128, 2003.

[24] M. Shur, P. Spalart, and M. Strelets, "Noise prediction for increasingly complex jets-part I: methods and tests," International Journal of Aeroacoustics, vol. 4, no. 3, pp. 213-246, 2005.

[25] C. Kato, A. Iida, Y. Takano, H. Fujita, and M. Ikegawa, "Numerical prediction of aerodynamic noise radiated from low Mach number turbulent wake," in Proceedings of the 31st Aerospace Sciences Meeting and Exhibit, Reno, Nev, USA, 1993.

[26] K. W. Chang, J. H. Seo, Y. J. Moon, and M. Roger, "Prediction of flat plate self-noise," in Proceedings of the 12th AIAA/CEAS Aeroacoustics Conference, pp. 1451-1464, May 2006.

[27] H. Yokoyama, K. Kitamiya, and A. Iida, "Flows around a cascade of flat plates with acoustic resonance," Physics of Fluids, vol. 25, no. 10, Article ID 106104, 2013.

[28] W. C. P. Van DerVelden, A. H. Van Zuijlen, A. T. De Jong, and H. Bijl, "On the estimation of Spanwise pressure coherence of a turbulent boundary layer over a flat plate," in Proceedings of 11th
World Congress on Computational Mechanics (WCCM '14), pp. 5710-5721, July 2014.

[29] J. W. S. Rayleigh, The Theory of Sound, Dover, Mineola, NY, USA, 1945.

[30] L. Larchevêque, P. Sagaut, I. Mary, O. Labbé, and P. Comte, "Large-eddy simulation of a compressible flow past a deep cavity," Physics of Fluids, vol. 15, no. 1, pp. 193-210, 2003.

[31] M. E. Goldstein, Aeroacoustics, McGraw-Hill, New York, NY, USA, 1976. 


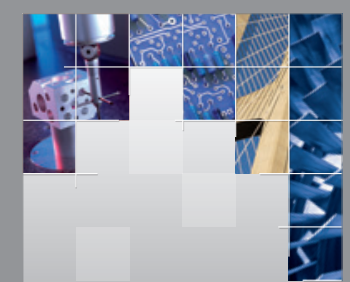

\section{Enfincering}
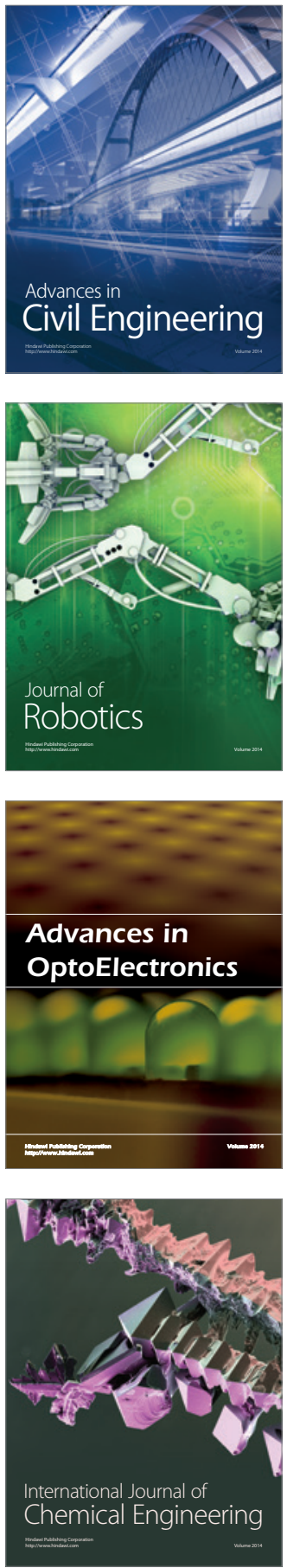

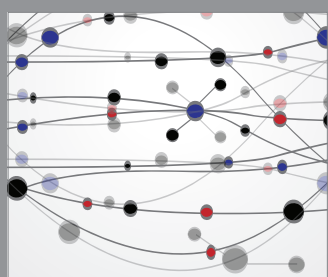

The Scientific World Journal

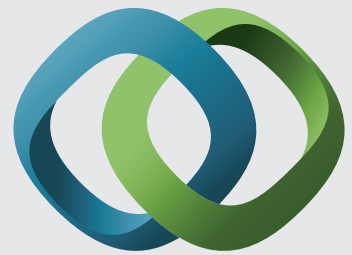

\section{Hindawi}

Submit your manuscripts at

http://www.hindawi.com
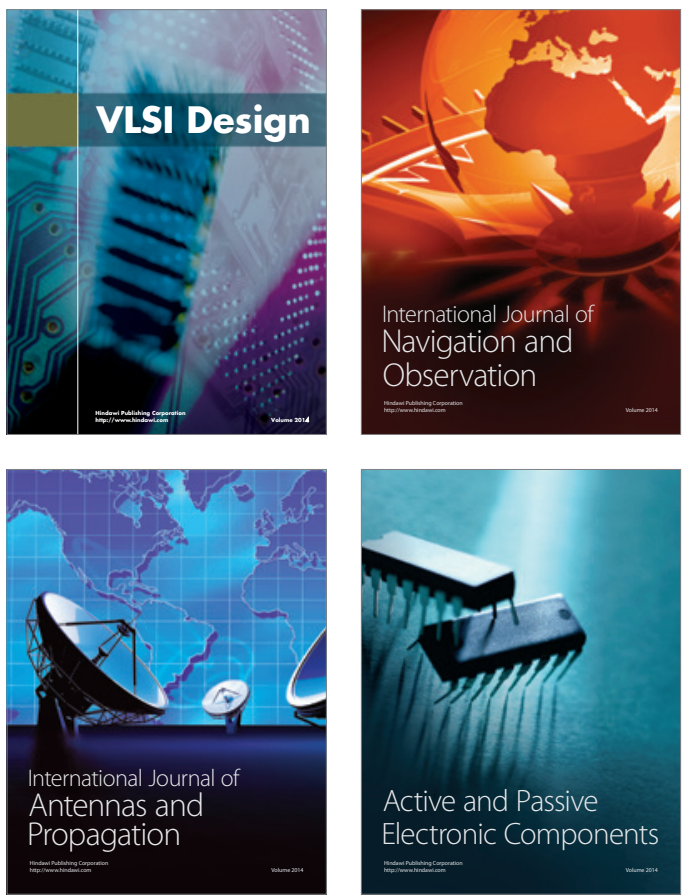
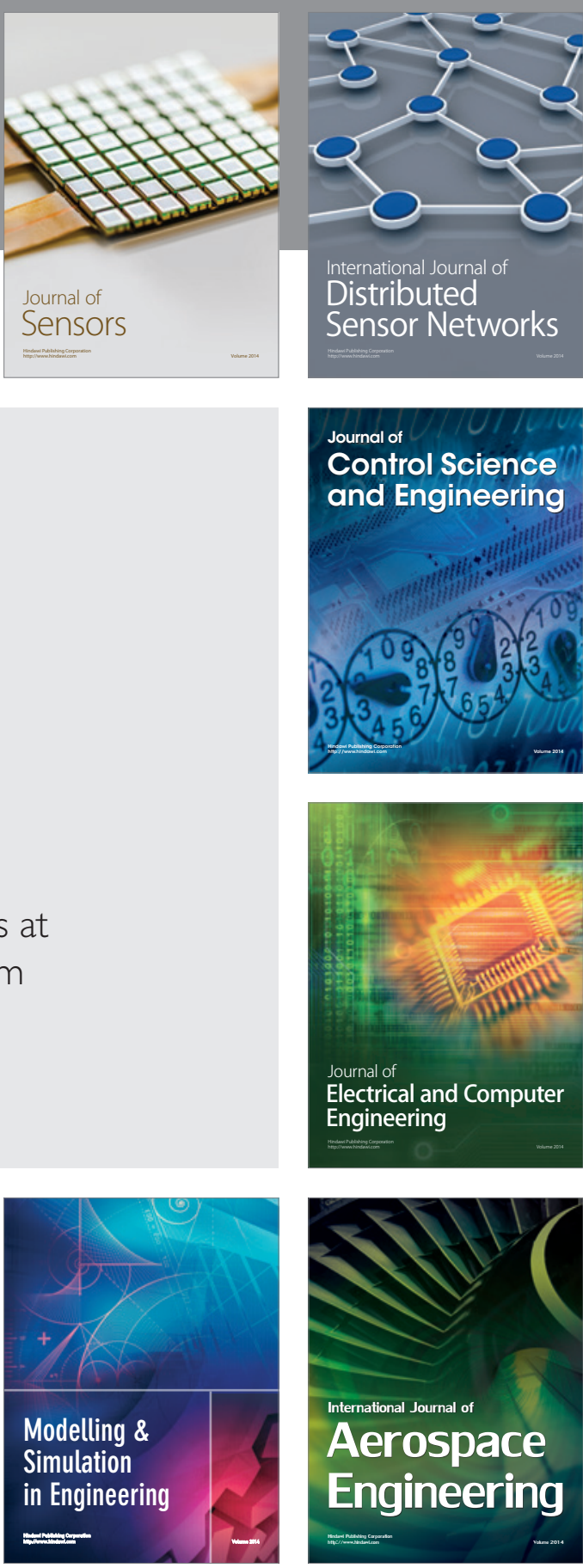

International Journal of

Distributed

Sensor Networks

Journal of

Control Science

and Engineering
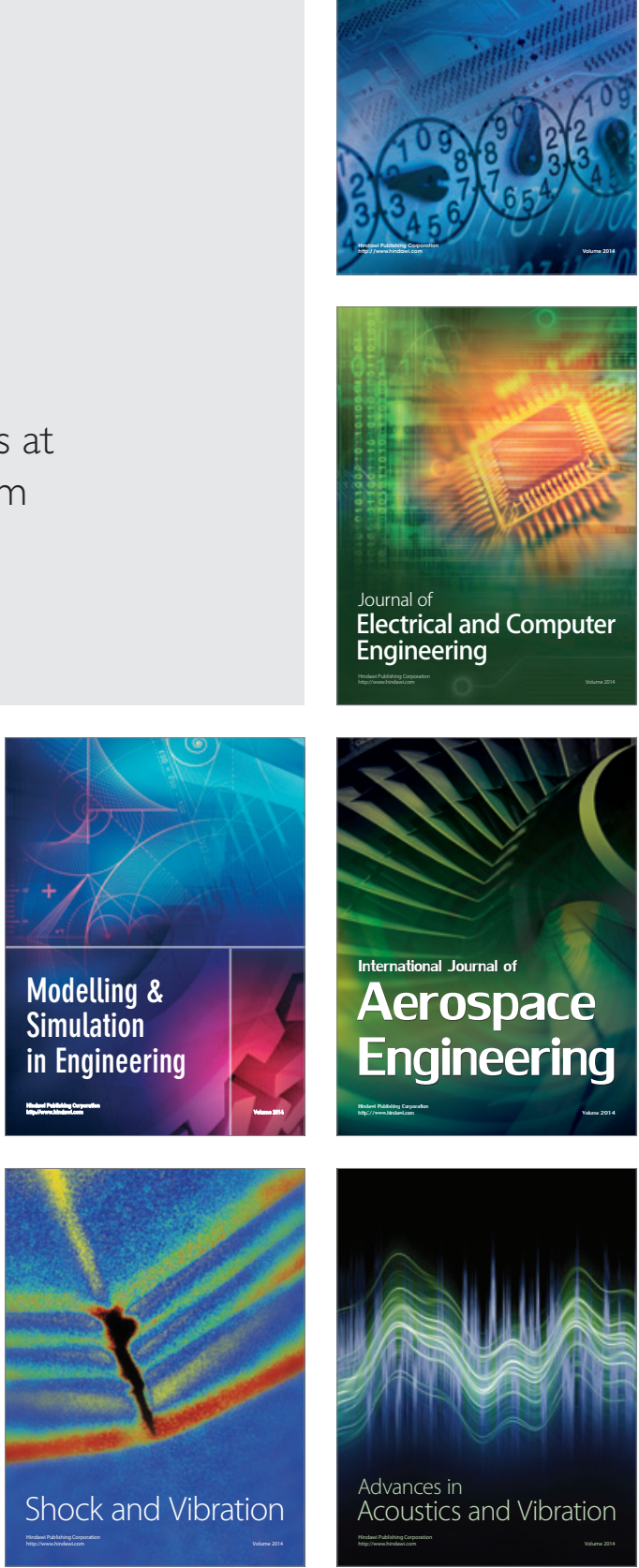